Review

\title{
Structure, inhibition, and regulation of essential lipid A enzymes
}

\author{
Pei Zhou* , Jinshi Zhao \\ Department of Biochemistry, Duke University Medical Center, Research Drive, DUMC 3711, Durham, NC 27710, USA
}

\section{A R T I C L E I N F O}

\section{Article history:}

Received 1 September 2016

Received in revised form 28 November 2016

Accepted 30 November 2016

Available online $\mathrm{xxxx}$

\begin{abstract}
A B S T R A C T
The Raetz pathway of lipid A biosynthesis plays a vital role in the survival and fitness of Gram-negative bacteria. Research efforts in the past three decades have identified individual enzymes of the pathway and have provided a mechanistic understanding of the action and regulation of these enzymes at the molecular level. This article reviews the discovery, biochemical and structural characterization, and regulation of the essential lipid A enzymes, as well as continued efforts to develop novel antibiotics against Gram-negative pathogens by targeting lipid A biosynthesis. This article is part of a Special Issue entitled: Bacterial Lipids edited by Russell E. Bishop
\end{abstract}

(c) 2016 Elsevier B.V. All rights reserved.

\section{Discovery of the Raetz pathway}

Over a century ago, Danish bacteriologist Hans Christian Gram devised a staining method that allowed the identification of Gramnegative bacteria from Gram-positive bacteria [1]. The molecular barrier that shielded the dye (crystal violet) from entering and staining the membrane of Gram-negative bacteria is now known as lipopolysaccharide (LPS). The attachment of LPS to the outer leaflet of the outer membrane of Gram-negative bacteria creates an asymmetric outer membrane with LPS in the outer leaflet and phospholipid in the inner leaflet. Such an asymmetric membrane provides an effective barrier to prevent harmful detergents and antibiotics from entering the cells.

LPS consists of three parts: an extended O-antigen chain, a core oligosaccharide domain, and a phosphorylated disaccharide lipid, known as lipid A. Lipid A serves not only as the hydrophobic membrane anchor of LPS, but also as the active component of the bacterial endotoxin, and displays strong modulatory effects of the human immune response.

The first complete chemical structure of lipid A, the species from Salmonella typhimurium, was elucidated by Takayama and colleagues in 1983 [2], revealing a hexaacylated disaccharide scaffold. Coinciding with elucidation of the lipid A structure was the discovery and structural elucidation of a novel Escherichia coli lipid, dubbed lipid X, that accumulates in E. coli mutants deficient in phosphatidylglycerol biosynthesis [3]. The chemical characterization of lipid X as 2,3-di-acylglucosamine 1-phosphate and the recognition of this lipid as a precursor of lipid $\mathrm{A}$ have enabled Christian R. H. Raetz and co-workers to propose a blueprint of the lipid A biosynthetic pathway [3-5], now known as the Raetz pathway, and the ultimate identification of individual enzymes of the pathway over a three-decade period (Fig. 1A,B) [6].

\footnotetext{
is This article is part of a Special Issue entitled: Bacterial Lipids edited by Russell E. Bishop. * Corresponding author.

E-mail address: peizhou@biochem.duke.edu (P. Zhou).
}

In E. coli, nine enzymes are required for the biosynthesis of $\mathrm{Kdo}_{2}-$ lipid A, which is sufficient to maintain the viability and fitness of virtually all Gram-negative bacteria [6]. After $\mathrm{Kdo}_{2}$-lipid $\mathrm{A}$ is synthesized in the cytosol, it is attached with core sugars and flipped out from the cytoplasmic surface of the inner membrane to the periplasmic surface, where it is further modified in some species of bacteria and transported to the outer membrane $[7,8]$.

Constitutive biosynthesis of lipid $A$ is required for the viability of nearly all Gram-negative bacteria. Although there exist a few exceptions, these strains do not occur naturally, and they display severely compromised fitness in the human host and enhanced sensitivity to a variety of antibiotics. For example, Chlamydia trachomatis, an obligate Gram-negative human pathogen, goes through a biphasic life cycle and transitions between the infectious elementary body (EB) form and the replicative reticulate body (RB) form during infection [9]. Chlamydia cells in the absence of constitutive lipid A biosynthesis are viable in the RB form, but are unable to transition back to the EB form, suggesting that lipid A may serve as a signaling molecule to coordinate the Chlamydia infection and pathogenesis [10]. Acinetobacter baumannii mutants devoid of lipid A biosynthetic genes have recently been isolated [11], though compounds disrupting lipid A biosynthesis still protected mice from lethal $A$. baumannii infection, indicating that the fitness of A. baumannii in the host environment is severely compromised [12]. Additionally, these mutant strains are hypersensitive to a variety of commercial antibiotics, including those that are only effective for Grampositive pathogens [13]. Finally, Neisseria meningitidis and Moraxella catarrhalis strains lacking lipid A biosynthesis have also been reported $[14,15]$. It remains to be seen whether the fitness and pathogenesis of these bacteria are similarly compromised in the human host as in the case of A. baumannii.

Of the nine lipid A enzymes in E. coli, the first six enzymes (LpxA, LpxC, LpxD, LpxH/I/G, LpxB, and LpxK) are essential, and their deletion cannot be rescued by compensatory mutations. As the lipid A 
(A)
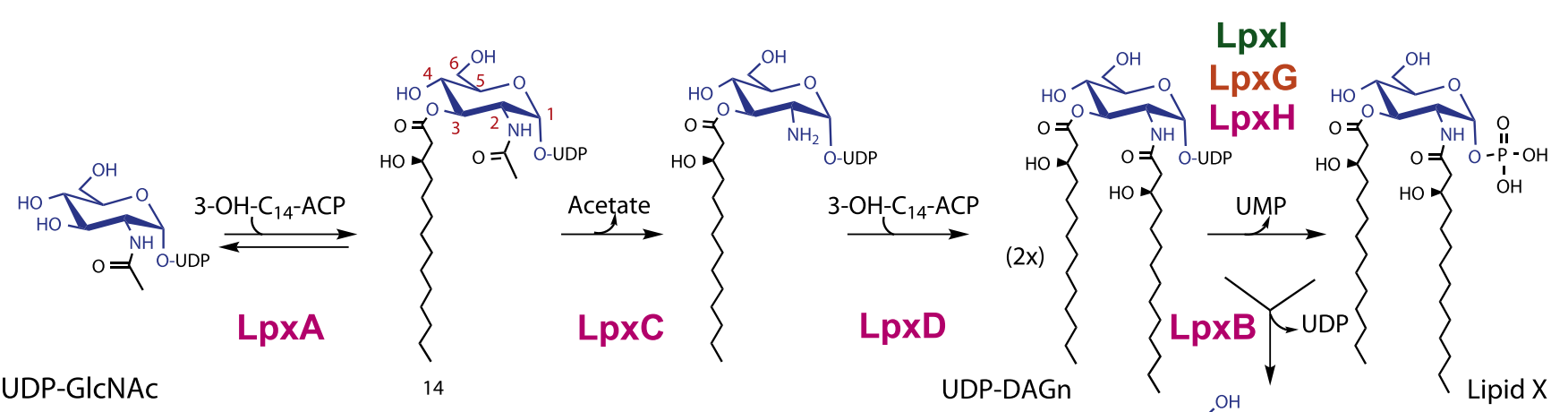

\section{Raetz Pathway}

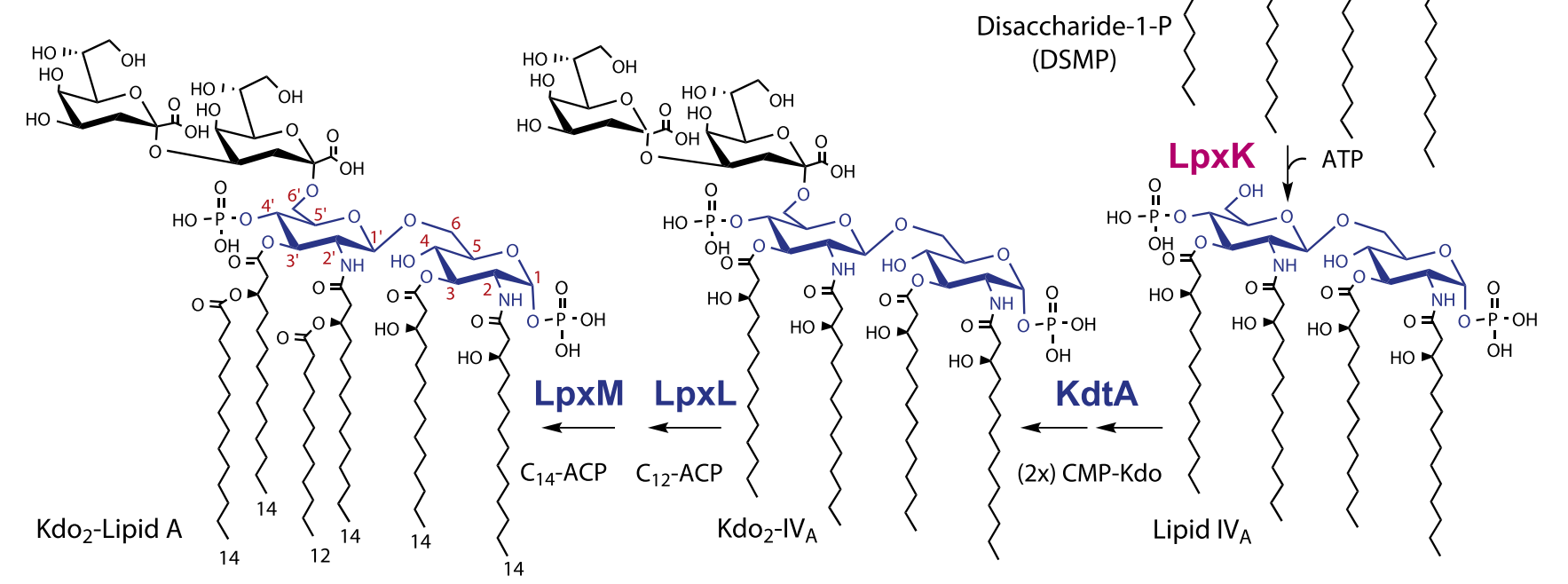

(B) initial proposal of

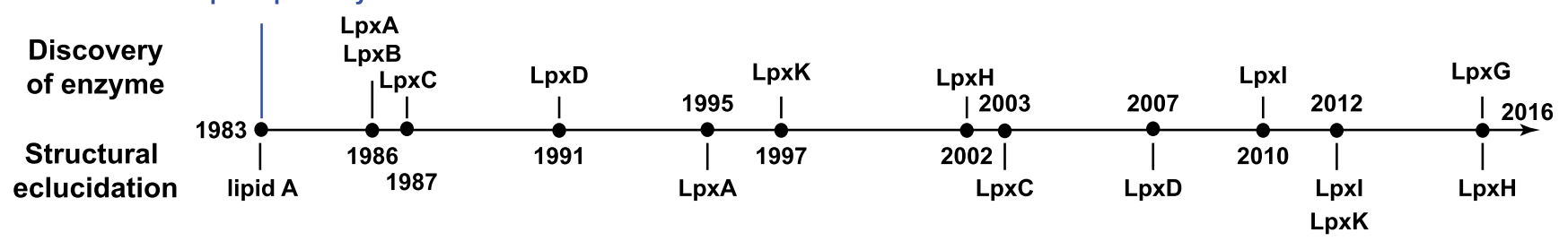

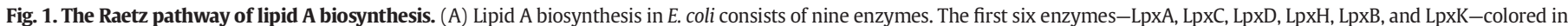

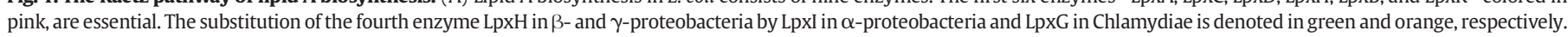

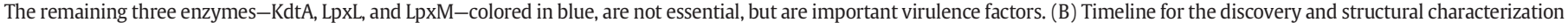
of essential lipid A enzymes.

biosynthetic pathway has never been exploited by commercial antibiotics, these essential lipid A enzymes are excellent targets for the development of novel antibiotics against Gram-negative pathogens to overcome established resistance mechanisms. The three remaining enzymes (KdtA, LpxL, and LpxM) are not essential, but they exert a profound effect on the fitness of the bacteria and may serve as antivirulence targets. Here, we review the discovery, biochemical characterization, and inhibition of essential lipid A enzymes and their regulation.

\section{LpxA}

LpxA is the first enzyme of the Raetz pathway (Fig. 1A). Incidentally, it is also the first lipid A enzyme that was molecularly cloned (together with LpxB, the fourth enzyme of the pathway, in 1986) [5] and structurally characterized [16] (Fig. 1B). With the exception of $C$. trachomatis LpxA, which utilizes myristoyl-ACP as the substrate [17], all other characterized LpxA enzymes catalyze the transfer of a $\beta$-hydroxyacyl chain from $\beta$-hydroxyacyl-ACP. In E. coli and the majority of Gram-negative bacteria, the $\beta$-hydroxyacyl chain is attached to UDP- $N$-acetyl-glucosamine (UDP-GlcNAc) at the 3-position via an ester bond. In this case, the acyl transfer reaction is thermodynamically unfavorable, with an estimated equilibrium constant of $\sim 0.01$ in E. coli [18]. It is important to note that not all of the LpxA-catalyzed acyl transfer reactions are unfavorable. A small set of Gram-negative bacteria, including Leptospira interrogans, synthesize a variant of lipid $A$, in which the glucosamine moiety is replaced with a 2,3-diamino-2,3-dideoxy-D-glucopyranose 
(GlcN3N) unit [19]. In this latter scenario, LpxA attaches the $\beta$ hydroxyacyl chain to the 3-position of GlcN3N through an amide bond instead of an ester bond, and the acylation reaction is thermodynamically favorable [20].

LpxA forms a biological homotrimer in solution, and its molecular architecture resembles a mushroom, with the central "stem" formed by three copies of a prism-shaped left-handed parallel $\beta$-helix domain at the N-terminus and the "cap" formed by a four-helix-bundle domain at the C-terminus (Fig. 2A). The left-handed parallel $\beta$-helix domain of LpxA consists of $\sim 30$ characteristic $\beta$-strands, with every three $\beta$ strands forming one complete coil of a $\beta$-helix in the shape of an equilateral triangle. In the middle of the $\beta$-helix domain exist two insertion loops that connect the subunit interfaces and form the pedestal of the active site for interaction with the UDP-GlcNAc substrate.
The structure of E. coli LpxA in complex with the product UDP-3-O( $R$-3-hydroxymyristoyl)-GlcNAc, shown in Fig. 2A, reveals a product at the interface of two adjacent LpxA subunits. The glucosamine ring is supported by the molecular pedestal formed by two insertion loops mentioned above. The 3-OH group of the glucosamine ring is buried, with its attached fatty acyl chain extending into the active site cleft along the subunit interface, whereas the UDP moiety extends toward the adjacent subunit to the right (Fig. 2A). The first four carbon atoms are almost perpendicular to the long axis of the $\beta$-helix. The rest of the hydrocarbon chain runs parallel to the $\beta$-helix.

LpxA enzymes are highly selective for the acyl chain length [21]. In E. coli and L. interrogans, LpxA prefers to incorporate 14-carbon and 12-carbon $R$-3-hydroxyacyl chains respectively [20,21], whereas Pseudomonas aeruginosa LpxA selects for an acyl chain of 10-carbon atoms

(A)
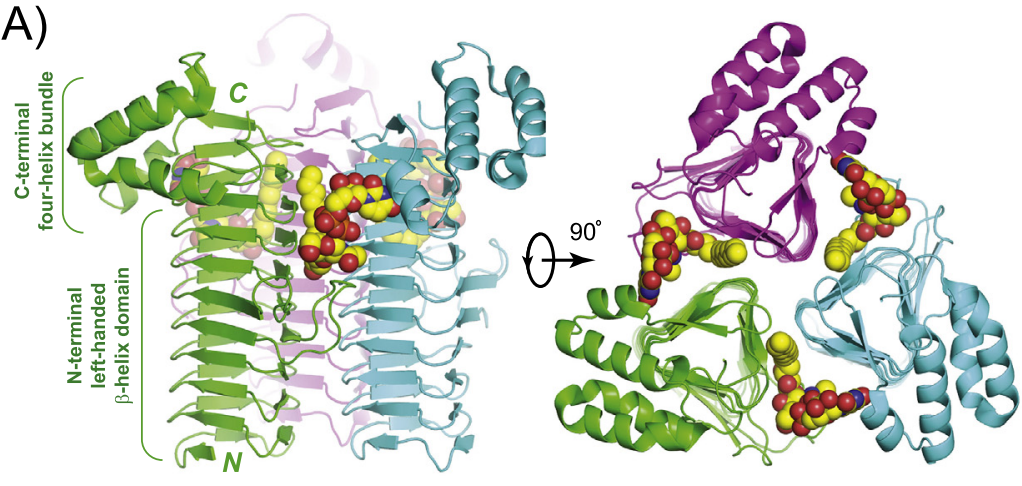

(C)

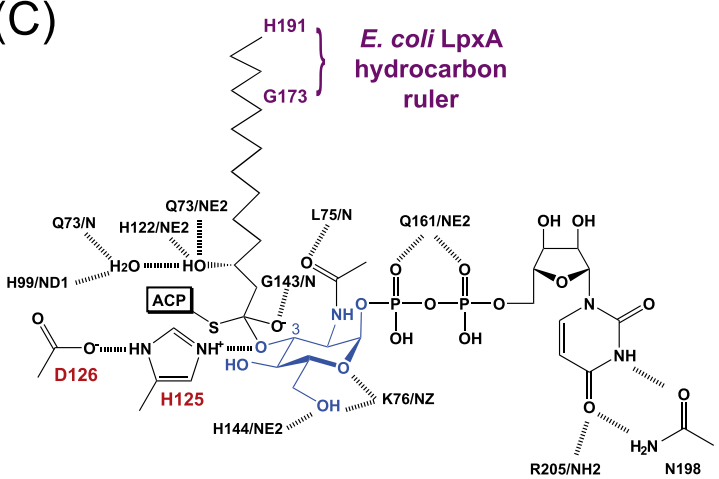

(B)

\section{E. coli LpxA}

\section{Leptospira LpxA}

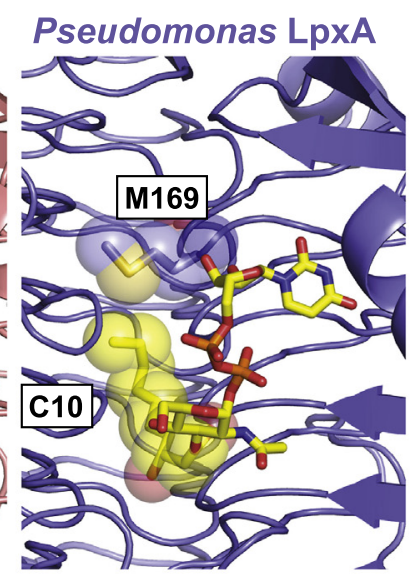

(D)
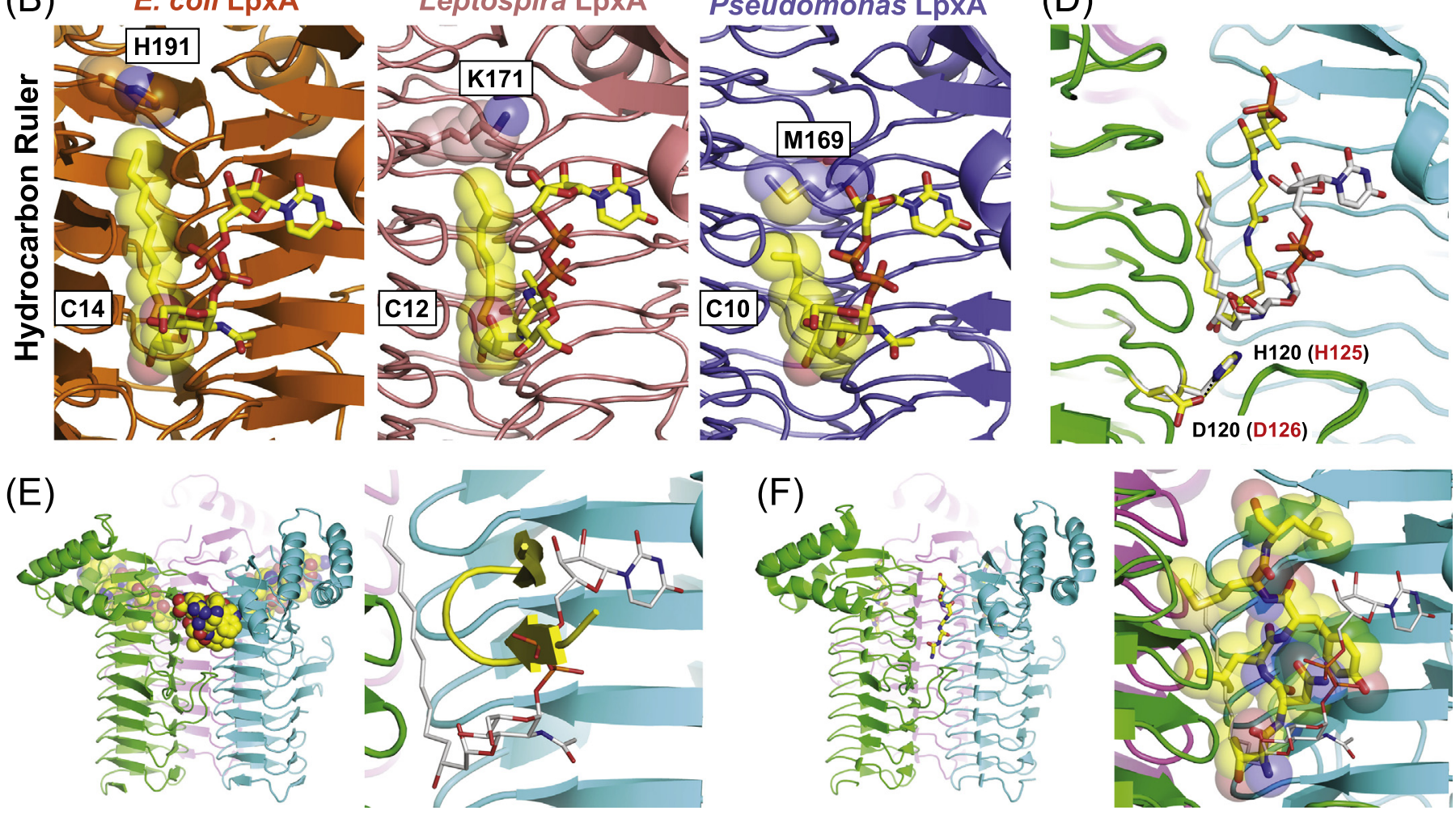

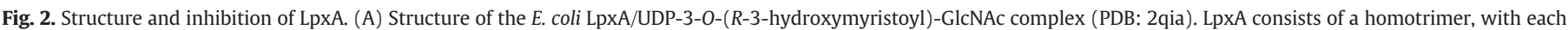

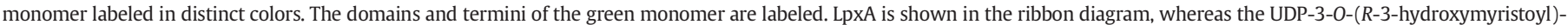

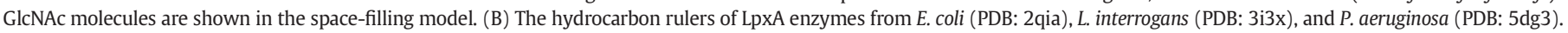

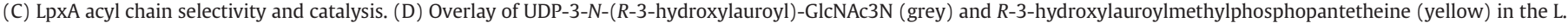

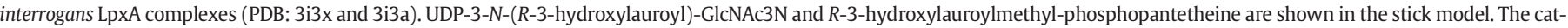

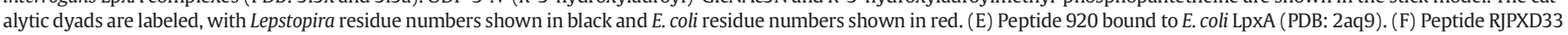
bound to E. coli LpxA (PDB: 4j09). Carbon atoms of peptides in panels (E) and (F) are colored in yellow, whereas carbon atoms of UDP-3-O-acyl-GlcNAc are colored in grey. 
(an R-3-hydroxydecanoyl chain) [21]. Structural analyses of the corresponding LpxA-product complexes have revealed the presence of a molecular ruler in all LpxA enzymes (Fig. 2B): in E. coli LpxA, along the hydrophobic cleft formed at the $\beta$-helix domain, H191 from the 10th $\beta$-coil blocks the further extension of the acyl chain and caps it to 14 carbons [22]; in L. interrogans LpxA, K171 from the 9th $\beta$-coil obstructs the hydrophobic cleft, restricting the length of the acyl chain to 12 carbons [20]; and in P. aeruginosa LpxA, M169 from a neighboring subunit jams the cleft even closer to the glucosamine ring, accommodating the acyl chain of only 10 carbon atoms [23]. Consistent with these structural observations, eliminating the steric blockage of M169 by a M169G mutation allows $P$. aeruginosa LpxA to preferentially incorporate a $\mathrm{C}_{14}$ acyl chain instead of a $C_{10}$ acyl chain, whereas introducing a steric block $(\mathrm{G} 173 \mathrm{M})$ in $E$. coli LpxA alters its preference from a $\mathrm{C}_{14}$ acyl chain toward a $\mathrm{C}_{10}$ acyl chain [21].

Biochemical studies of $E$. coli LpxA suggested a mechanism in which $\mathrm{H} 125$ in the middle of the central $\beta$-helix serves as the catalytic base to active the 3-OH group of the substrate UDP-GlcNAc, which in turn attacks the thioester bond of the second substrate, acyl-ACP to replace ACP (Fig. 2C) [24]. Such a mechanism is supported by the observation of a hydrogen bond between $\mathrm{H} 125$ and the 3-O atom of the glucosamine ring of UDP-3-O-acyl-GlcNAc in complex with E. coli LpxA [22]. The structures of $L$. interrogans LpxA in complex with UDP-3- $N$-( $R$-3-hydroxylauroyl)GlcNAc3N and with $R$-3-hydroxylauroylmethylphosphopantetheine, a mimetic of acyl-ACP, have also been determined [20]. In the acylpantetheine complex, the acyl chain and the pantetheine arm of the acyl-ACP form a tight U-turn, with the pantetheine arm packing antiparallely with the acyl chain. Notably, the acyl chain of $R-3-$ hydroxylauroylmethylphosphopantetheine nearly completely overlaps with that of UDP-3- $N$-( $R$-3-hydroxylauroyl)-GlcNAc3N, with the pantetheine group siting between the UDP-GlcNAc3N moiety and the acyl chain of UDP-3-N-(R-3-hydroxylauroyl)-GlcNAc3N (Fig. 2D). Such a structural observation strongly suggests an ordered bi-substrate mechanism for LpxA: the binding of acyl-ACP occurs first, which is followed by the binding of UDP-GlcNAc/UDP-GlcNAc3N; subsequently the acyl chain transfer occurs via a substrate-assisted mechanism facilitated by the catalytic histidine residue (e.g., H125 in E. coli).

Potent peptide inhibitors of LpxA have been reported [25,26]. Peptide 920 , discovered through a phage-display library screening, inhibited bacterial growth when it was overexpressed in cells as a GST-fusion protein [27]. Using enzyme kinetics analysis, Williams and co-workers determined that peptide 920 competes with acyl-ACP for LpxA binding, with a $K_{\mathrm{i}}$ value of $\sim 50 \mathrm{nM}$ [25]. Structural analysis shows that peptide 920 adopts a $\beta$-hairpin conformation and binds to the deep cleft at the LpxA trimer interface near the active site (Fig. 2E) [25]. Interestingly, peptide 920 not only occupies the space of the pantetheine group of the acyl-ACP analog, acyl-phosphopantetheine [20], but also overlaps in space with the substrate UDP-GlcNAc and the UDP-GlcNAc moiety of the product UDP-3-O-acyl-GlcNAc (Fig. 2E). Hence, the observation that peptide 920 is a competitive inhibitor against acyl-ACP, but not UDP-GlcNAc, is consistent with an ordered sequential bi-substrate binding process, in which acyl-ACP binds LpxA first, and UDP-GlcNAc binds second. Due to its membrane impermeability, peptide 920 has no measurable antibiotic activity in vitro. However, its compact hairpin conformation may provide an attractive starting scaffold for the design of cyclic peptide analogs [28] and modified polyketides [29] as cell-permeable antibiotics.

RJPXD33 is a dual-specific peptide inhibitor of LpxA and LpxD, the third enzyme of the pathway [26]. It is a weaker inhibitor of LpxA compared to peptide 920 , with an estimated $K_{\mathrm{d}}$ of $20 \mu \mathrm{M}$ for $E$. coli LpxA. The structure of RJPXD33 was captured in complex with E. coli LpXA (Fig. 2F), revealing an extended conformation that is distinct from the hairpin structure of peptide 920 [30]. The peptide backbone of RJPXD33 runs parallel with the acyl chain of the UDP-3-O-acyl-GlcNAc molecule, with its amino acid sidechains reaching out to the space occupied by the acyl chain and the UDP-GlcNAc moiety (Fig. 2F). Despite the relatively weak binding affinity of RJPXD33 and the lack of antibiotic activity in vitro, it remains an attractive lead scaffold for further optimization of peptidomimetics due to its dual specificity against two essential enzymes in the lipid A pathway.

\section{LpxC}

The sequence of the envA gene that encodes LpxC, the second enzyme of the Raetz pathway (Fig. 1A), was decoded in 1987 [31], shortly after the molecular cloning of LpxA and LpxB. Although envA was shown as an essential gene involved in the maintenance of bacterial membrane integrity, its biological function as the UDP-3-O-acyl-GlcNAc deacetylase in lipid A biosynthesis was not deciphered until 1995 [32]. The envA gene was subsequently renamed as $l p x C$.

As the acylation reaction catalyzed by LpxA is thermodynamically unfavorable for most Gram-negative bacteria [18], the deacetylation reaction catalyzed by LpxC is thought to be the committed step of lipid A biosynthesis [33]. LpxC is a metal-dependent amidase [34-36]. It is highly active in the presence of $\mathrm{Zn}^{2+}$ ions [34] and has a dissociation constant $\left(\mathrm{K}_{\mathrm{d}}\right)$ of $\sim 60 \mathrm{pM}[35]$. $\mathrm{Zn}^{2+}$ is also the only significant ion that co-purifies with LpxC from E. coli grown in rich media [34]. Therefore, LpxC is widely acknowledged as a $\mathrm{Zn}^{2+}$-metalloamidase [34]. However, LpxC is 6- to 8-fold more active in the presence of the oxygen-sensitive $\mathrm{Fe}^{2+}$ cofactor [36]. Although LpxC's affinity for $\mathrm{Fe}^{2+}$ is much weaker, with a $K_{d}$ value of $\sim 110 \mathrm{nM}$, the metal bound to purified LpxC from E. coli grown in the minimal medium is mainly $\mathrm{Fe}^{2+}$ [35]. Thus, LpxC may utilize both $\mathrm{Zn}^{2+}$ and $\mathrm{Fe}^{2+}$ in vivo and can switch between these two metal ions in response to the change of the environment and redox potential [35]. Due to the early identification of $\mathrm{LpxC}$ as a $\mathrm{Zn}^{2+}$ dependent enzyme, the high affinity of LpxC toward the $\mathrm{Zn}^{2+}$ ion, and the stability of the $\mathrm{Zn}^{2+}$ ion exposed to air, all of the structural analyses of LpxC thus far have been conducted using $\mathrm{Zn}^{2+}$ as the metal cofactor.

The first structures of LpxC were reported in 2003, one in complex with a substrate analog inhibitor TU-514 by solution NMR [37] and one in complex with a myristate by crystallography [38]. The solution structure of Aquifex aeolicus LpxC in complex with the substrate analog TU-514, shown in Fig. 3A, reveals a novel protein fold consisting of two domains. Each domain contains a layer of $\alpha$-helices packing against a layer of $\beta$-sheet. The two domains come together to form a "sandwich" by packing helices in the interior and $\beta$-sheets on the outside. Each domain also contains a unique insert region. Insert I of domain I consists of a small $\beta$-sheet with three short $\beta$-strands, whereas Insert II of domain II consists of a $\beta-\alpha-\beta$ structure that forms a hydrophobic passage to accommodate the acyl chain of the substrate and the substrate analog, TU-514.

The active site of $A$. aeolicus LpxC in complex with the product, UDP3-O-acyl-glucosamine (UDP-3-O-acyl-GlcN), is shown in Fig. 3B [39]. It is located between the two domains, with the catalytic $\mathrm{Zn}^{2+}$ ion $\left(\mathrm{Zn}_{\mathrm{A}}\right)$ coordinated by two His (H74 and H226) and one Asp (D230) residues. An inhibitory $\mathrm{Zn}^{2+}$ ion $\left(\mathrm{Zn}_{\mathrm{B}}\right)$ is also present in the crystal structure and is in part coordinated by the catalytically important residues E73 and $\mathrm{H} 253$. The glucosamine ring sits near the catalytic $\mathrm{Zn}^{2+}$ ion, and the UDP moiety extends out across the active site to reach a solventexposed surface area of domain II. K227, an essential residue for catalysis, plays an important role in bridging the pyrophosphate group of the UDP moiety.

Several models of LpxC catalysis have been proposed based on metal substitution, mutagenesis, structural, and $\mathrm{pH}$ dependence studies [37-44]. The emerging view implicates E73 as the catalytic general base for deprotonation of the catalytic $\mathrm{Zn}^{2+}$-bound water (Fig. 3C). The oxyanion of the tetrahedral transition state is proposed to be stabilized by the hydroxyl group of T191. H253, which has an elevated pKa and is protonated at neutral $\mathrm{pH}$ [40], likely donates a proton to the product amine group to complete the catalysis. Interestingly, these studies have also found that E. coli LpxC mutants with E78A (E73 in A. aeolicus LpxC) or H258A (H253 in A. aeolicus LpxC) retained a bell-shaped pH 


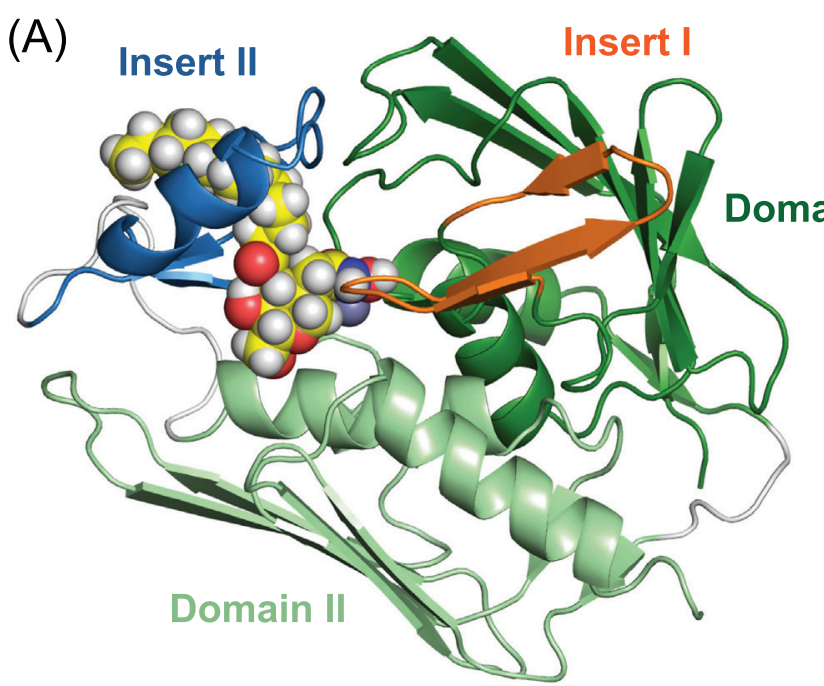

(C)

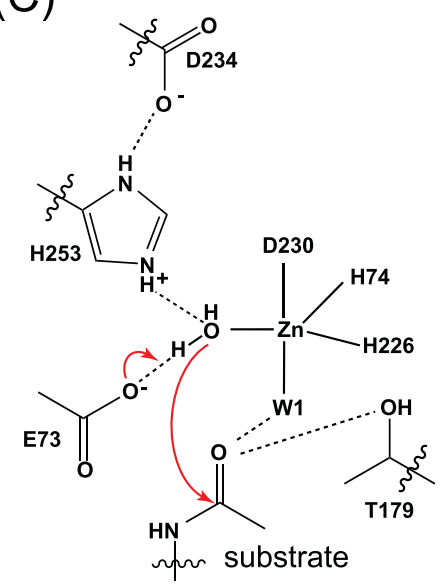

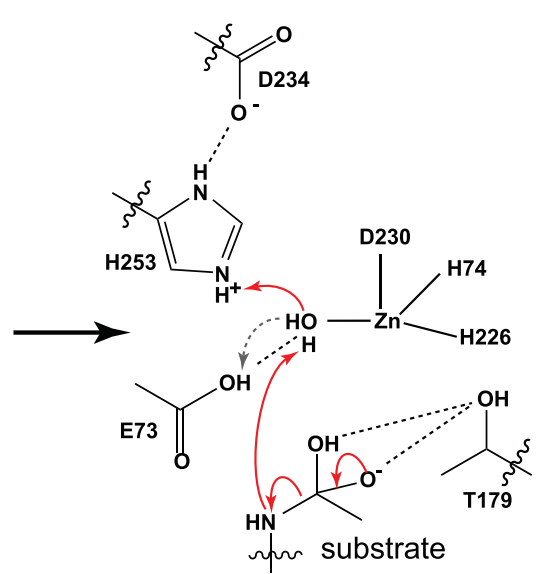

(E)

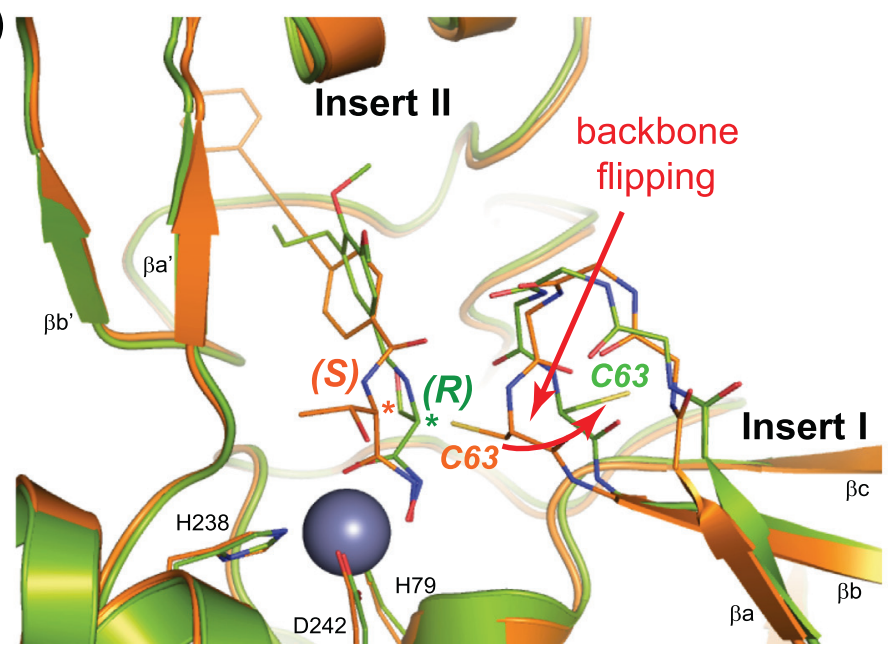

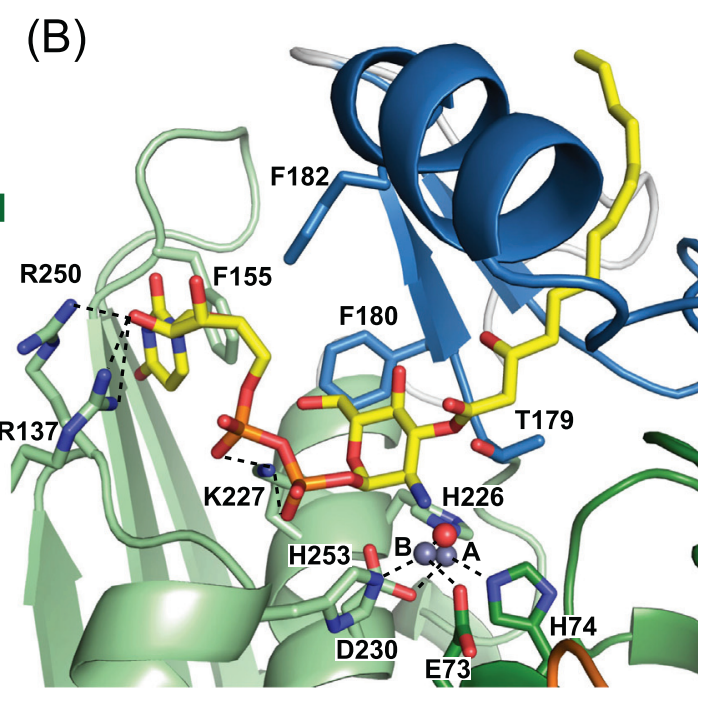

(D) $\frac{\text { LpxC inhibitors }}{\text { tail group }} \frac{\text { head group }}{\text { heula }}$<smiles>CCCc1cc(C2=NC(C(=O)NO)CO2)cc(OC)c1OC</smiles><smiles>[R]/[R9]=C/C</smiles><smiles>[R]c1ccc(C#CC#Cc2ccc(C(=O)NC(C(=O)NO)C(C)O)cc2)cc1</smiles><smiles>[R]CC=CC</smiles><smiles>COc1ccc(-c2ccn(CCC(C(C)=O)(C(=O)O)C(=O)NO)c(=O)c2)c(F)c1</smiles>

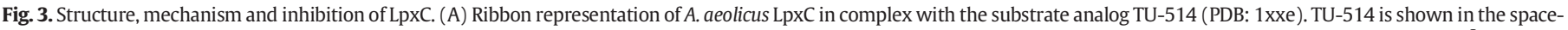

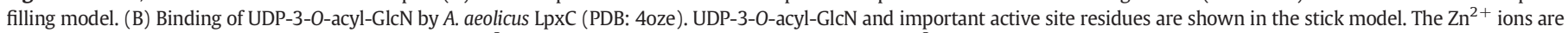

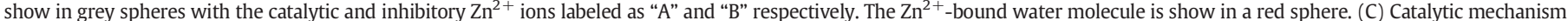

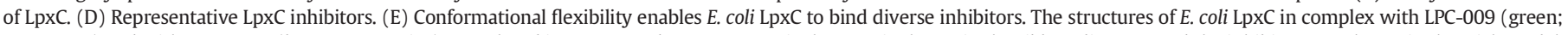

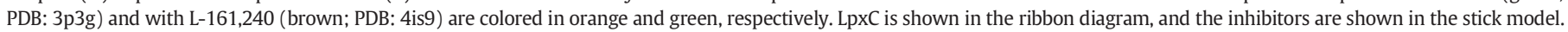

rate profile, albeit at 100- to 1000 -fold reduced levels of activity compared with the wild-type enzyme [42], suggesting the existence of a compensatory mechanism. A possible solution to this dilemma may involve an additional water molecule that bridges both E73 and $\mathrm{H} 253$ for catalysis and proton shuffling, such that either E73 or H253 could function as the water activator or proton donor in the absence of the other residue (Fig. 3C) [39]. If this is true, the bell-shaped $\mathrm{pH}$ rate profile of LpxC may reflect the pKa values of E73 and this $\mathrm{Zn}^{2+}$-bound water 
molecule [41,42], rather than those of E73 and H253. Further structural analysis of a substrate complex or a transition state analog complex will be invaluable for understanding the full details of the catalytic mechanism of LpxC.

Shortly after deciphering the biological function of LpxC in lipid A biosynthesis, Onishi and colleagues reported the discovery of a phenyl-oxazoline hydroxamate based LpxC inhibitor, L-161,240, that inhibited E. coli LpxC with an $\mathrm{K}_{\mathrm{i}}$ of $\sim 50 \mathrm{nM}$ (Fig. 3D) [45]. This compound not only displayed impressive antibiotic activity against $E$. coli and closely related bacteria in vitro, but also cured mice infected with a lethal intraperitoneal dose of $E$. coli. A major limitation of L-161,240 is its narrow spectrum of antibiotic activity. Notably, it is inactive against $P$. aeruginosa. Such a lack of activity against $P$. aeruginosa LpxC appeared to be caused by the rigidity of the $P$. aeruginosa enzyme, whereas the E. coli enzyme is conformationally dynamic and can undergo a conformational switch that expands the active site to accommodate inhibitors with various head groups, including compounds with opposite stereo centers at the $C \alpha$ position next to the hydroxamate group [46] (Fig. 3E). The subsequent discovery of CHIR-090 by Chiron and University of Washington was a game changer for the development of LpxC inhibitors [47], as this compound was active against both WT E. coli and P. aeruginosa [48]. CHIR-090 consists of a hydroxamate-amino acid head group coupled to a morpholine biphenyl acetylene tail group. Replacing the acetylene group of CHIR-090 with a bisacetylene group, such as in LPC-009/011, broadens the spectrum of antibiotic activity [49,50]. Structural analysis of LpxC in complex with LPC-009 shows that the amino acid moiety of LPC-009 is engaged in specific interactions with conserved residues of LpxC in the active site; the hydroxamate group chelates the catalytic $\mathrm{Zn}^{2+}$ ion; and the tail group penetrates through the LpxC hydrophobic passage harboring the acyl chain of the substrate (Fig. 3E). Another representative compound, PF-5081090 [51], which features a pyridone methylsulfone hydroxamate scaffold (Fig. 3D), also displays excellent antibiotic activity in vitro and in murine infection models [52]. The most potent LpxC inhibitor in vitro documented to date is LPC-058, which is a time-dependent inhibitor with a $\mathrm{K}_{\mathrm{i}}^{*}$ value of $3.5 \pm$ $0.2 \mathrm{pM}$ for the tight, slow forming E. coli LpxC-inhibitor complex and displays a broad range of activity against E. coli, P. aeruginosa, S. typhimurium, Vibrio cholerae, Klebsiella pneumoniae, Enterobacter cloacae, Morganella morganii, Proteus mirabilis, C. trachomatis, A. baumannii, and clinical isolates [53,54]. A common feature of these inhibitors is that they share a hydroxamate-containing head group that chelates the catalytic $\mathrm{Zn}^{2+}$ ion in the active site and a greasy tail group that occupies the hydrophobic acyl-chain binding passage (Fig. 3D).

\section{LpxD}

The gene encoding LpxD, the third enzyme of the lipid A pathway (Fig. 1A), was cloned and sequenced in 1991, though it was first named firA for its role in reversal of certain rifampin-resistant mutations [55] and was thought to play a role in transcription [56]. Based on its significant sequence homology to LpxA and its location within the same complex operon as $\operatorname{lp} x A$ and $\operatorname{lp} x B$ in $E$. coli, the firA gene product was tested for its possible participation in lipid A biosynthesis. Such efforts led to the biochemical characterization of firA in 1993 as $\operatorname{lp} x D$, the gene encoding the second acyl transferase to convert UDP-3-O-acylGlcN to UDP-2,3-diacylglucosamine (UDP-DAGn) at the third step of the Raetz pathway [57].

The first structure of LpxD, the ortholog from C. trachomatis, was reported in 2007 [58], though the topological arrangement of its Cterminal domain is distinctly different from all other LpxD ortholog structures from E. coli [59], P. aeruginosa [60] and A. baumannii [61]. The structure E. coli LpxD, shown in Fig. 4A, shares a common domain arrangement with LpxD orthologs from $P$. aeruginosa and A. baumannii, and possesses an intertwined homotrimic structure with a characteristic central prism of the left-handed $\beta$-turn helix similar to that seen in LpxA. Each monomer also contains an N-terminal uridinebinding domain and a C-terminal helix domain that engages ACP.

Steady-state enzyme kinetics analysis suggests that LpxD utilizes a substrate-assisted catalytic mechanism [62]. H239 located in the middle of the left-handed $\beta$-turn helix of LpxD serves as the catalytic base to deprotonate the 2-amino group of the glucosamine ring of UDP-3-Oacyl-GlcN, which then attacks the carbonyl carbon of the thiol ester bond of $\beta$-hydroxyacyl-ACP [62], with the oxyanion being stabilized by the backbone of G257 (Fig. 4B). LpxD is also highly selective for the acyl chain length [59]. E. coli LpxD prefers to transfer an acyl chain of 14 carbon atoms over a chain of 16 carbon atoms at a ratio of 3:1. Such selectivity is caused by the steric blockage of the acyl chain binding grove by a bulky residue, M290, in E. coli LpxD (Fig. 4B). Removal of the steric blockage by the M290A mutation alters the selectivity of $E$. coli LpxD toward a 16-carbon unit acyl chain [59].

The LpxD reaction undergoes a compulsory ordered bi-substrate mechanism (Fig. 4C) [62]. In such a mechanism, $R-3-\mathrm{OH}-\mathrm{C}_{14}-\mathrm{ACP}$ (acyl-ACP) binds LpxD first to form the EA complex; this is followed by the binding of UDP-3-O- $\left(R-3-\mathrm{OH}-\mathrm{C}_{14}\right)-\mathrm{GlcN}$ to form the bisubstrate complex $E A B$; the acyl chain transfer yields the bi-product complex EPQ; one of the products, UDP-DAGn, dissociates first, leaving behind the LpxD-holoACP complex (EQ); finally, the holoACP departs to regenerate LpxD. The tight affinities between various forms of ACP and LpxD have enabled structural snapshots of the intermediates of the LpxD/acyl-ACP complex (EA), the LpxD/holo-ACP (EQ) complex, as well as a hydrolyzed acyl-ACP LpxD complex that resembles the postcatalysis EPQ complex during the catalytic cycle (Fig. 4C) [63]. In all of these structural states, the ACP docks on top of the C-terminal ACPrecognition domain formed by the trimeric $C$-terminal helical domain and the last $\beta$-coil of the left-handed $\beta$-helix domain (Fig. 4D).

In the acyl-ACP complex bound to the catalytically inactive LpxD (H253A) (Fig. 4C, left, EA state), acyl-ACP has its prosthetic group and acyl chain swinging out of the ACP hydrophobic chamber and forming extensive contacts with LpxD. This is in sharp contrast with free ACP, which has its acyl chain encapsulated within the hydrophobic chamber [64]. The $\beta-\mathrm{OH}-\mathrm{C}_{14}$ acyl chain and the $4^{\prime}$-phosphopantetheine (4'-PPT) arm together adopt a horseshoe-like conformation, with the acyl chain (denoted as the "N-linked" acyl chain to indicate the location of the 2$N$ acyl chain of the product) buried in a hydrophobic channel of LpxD. The 4'-PPT arm extends over $14 \AA$ from ACP to reach the alaninesubstituted $\mathrm{H} 239$ catalytic base in the middle of the left-handed $\beta$ helix (Fig. $4 \mathrm{C}$, left, EA state). The terminal carbon atoms of $\beta-\mathrm{OH}-\mathrm{C}_{14}$ pack against the "hydrocarbon ruler" M290 located at the far end of the $N$-channel (Fig. 4C, inset).

In the hydrolyzed acyl-ACP structure (Fig. 4C, middle, EPQ state), two $\beta-O H-C_{14}$ fatty acid molecules were bound to the LpxD surface: one reflects the hydrolyzed acyl chain from acyl-ACP ( $N$-linked), whereas the second one occupies a separate hydrophobic channel ( $O$-linked) that accommodates the acyl chain of the substrate, UDP-3-O-acyl-GlcN.

Most interestingly, the structure of the holo-ACP complex (Fig. 4C, right, $E Q$ state) reveals a substantial movement of the PPT arm. Notably, the terminal thiol group of the 4'-PPT has vacated the catalytic cleft and moved $\sim 15 \AA$ to be situated near M290 (Fig. 4C inset), propelling the UDP-DAGn product to dissociate before the release of holo-ACP.

Like the two previous enzymes in the Raetz pathway, LpxD is an excellent antibiotic target. Using a phage display library, Jenkins and Dotson discovered a peptide inhibitor that simultaneously inhibits LpxD and LpxA [26]. Structural elucidation of the corresponding LpxDpeptide complex may provide important insights to design LpxDtargeting antibiotics.

\section{LpxH, LpxI, and LpxG}

In contrast to other steps of lipid A biosynthesis, each of which is carried out by a single enzyme conserved throughout Gram-negative bacteria, the fourth step and the first membrane-associated step of the 


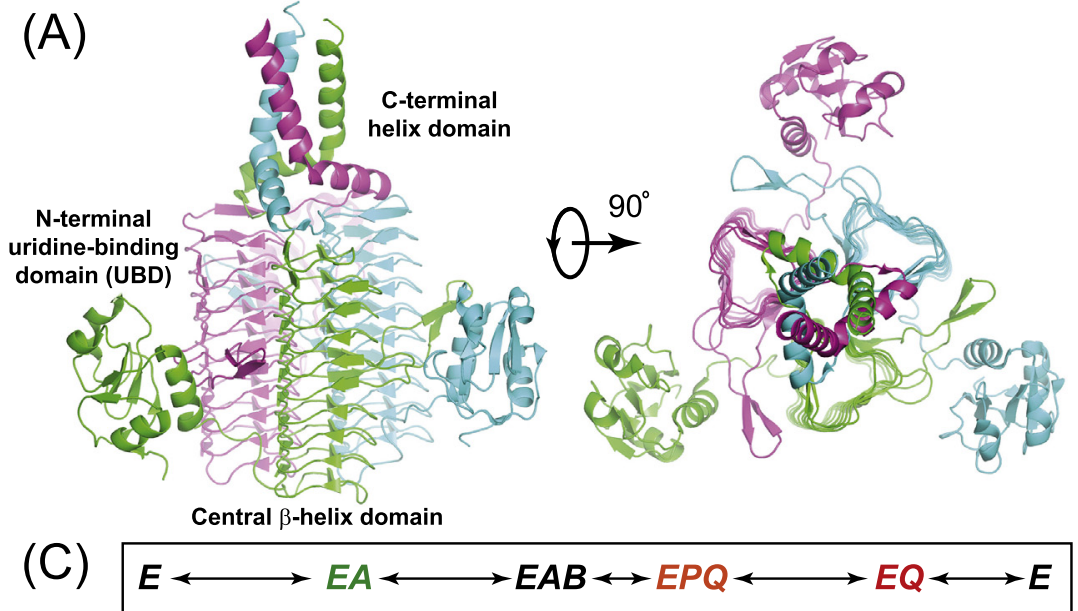

Acyl-ACP Hydrolyzed-Acyl-ACP Holo-ACP

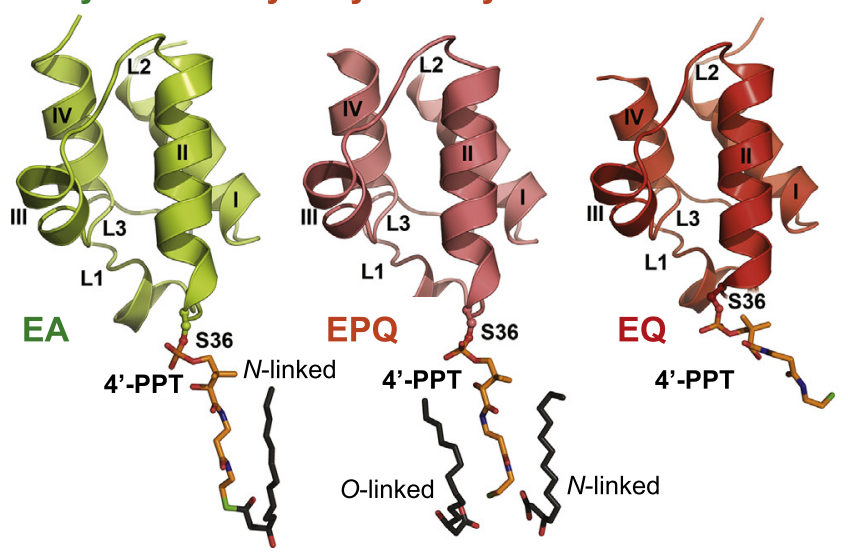

(B)
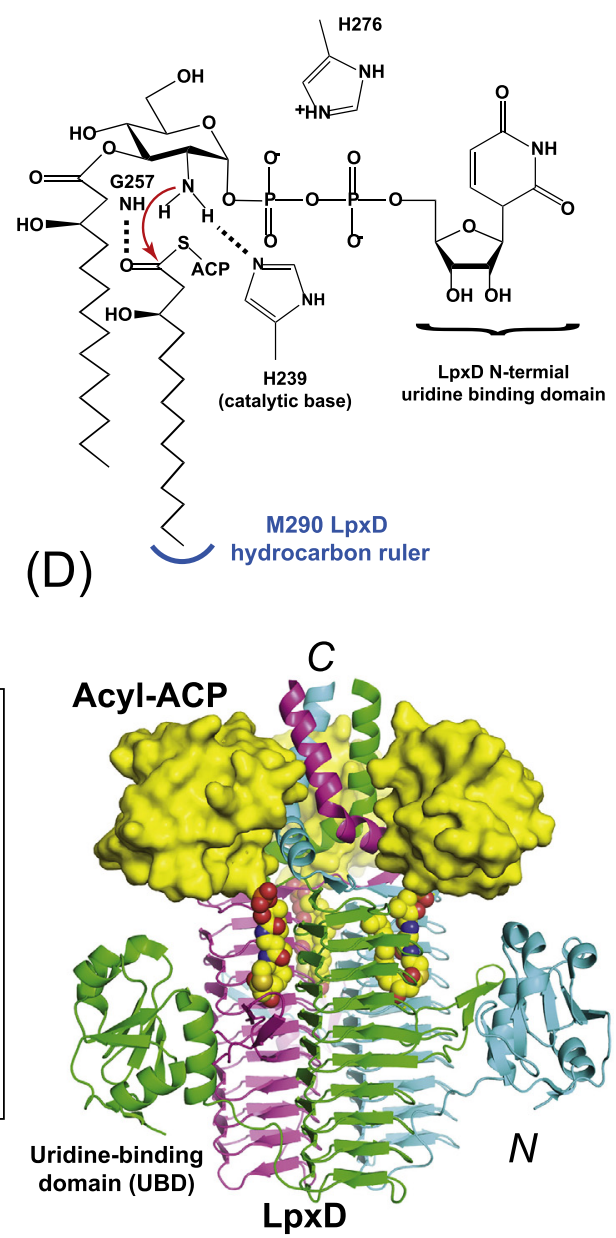

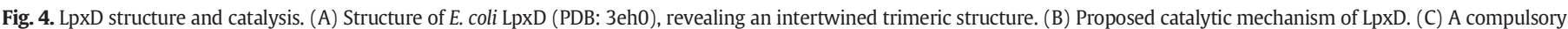

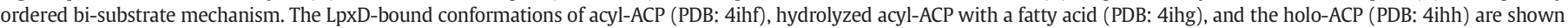

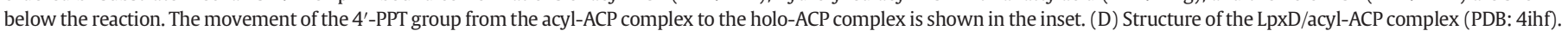

pathway, the cleavage of the pyrophosphate group of UDP-DAGn to form lipid X, is carried out by LpxH in $\beta$ - and $\gamma$-proteobacteria [65], LpxI in $\alpha$-proteobacteria [66], and LpxG in Chlamydiae [67] (Fig. 1A). These enzymes do not co-exist in the same organism. Among these functional orthologs, LpxH is most widespread, occurring in $~ 70 \%$ of Gram-negative bacteria, including major Gram-negative pathogens. LpxI exist in 30\% of the Gram-negative bacteria, whereas the distribution of LpxG is much limited, only existing in Chlamydiae [67] and, based on sequence similarity, Synechococcus.

\section{1. $\mathrm{LpxH}$}

The expression cloning of the UDP-DAGn pyrophosphate hydrolase was confounded by the presence of the background activity of CDPdiglyceride hydrolase (Cdh) in E. coli [68], which catalyzes the same hydrolysis reaction in vitro, but does not contribute to lipid A biosynthesis in vivo $[68,69]$. Consequently, the $l p x H$ gene was identified using a $c d h$ deficient $E$. coli strain and the expression cloning of the Kohara library $[65,70]$. Such efforts led to the identification of $y b b F$ in $E$. coli, a gene encoding a protein of unknown function as $l p x H$ in 2002 [65]. Biochemical characterization shows that E. coli LpxH is a member of the calcineurin-like phosphoesterases (CLPs), requires detergent for full activity, and is an essential enzyme [65,71]. Further studies using the more stable Haemophilus influenzae LpxH ortholog show that LpxH retains full activity in the presence of $\mathrm{Mn}^{2+}$, but not other metal ions, and utilizes a di-manganese cluster for catalysis [72].
The structure of $H$. influenzae LpxH in complex with the product, lipid X, was recently determined [73], revealing a unique insertion lid above the conserved core architecture of calcineurin-like phosphoesterases (Fig. 5A). The active site is formed between the insertion lid and the core CLP domain. In the active site, a di-manganese cluster is chelated by residues of the signature metal chelating motifs of the CLP enzymes (Fig. 5B). Alanine substitutions of these metal chelating residues significantly reduced the activity of $H$. influenzae LpxH [72]. The glucosamine-1-phosphate head group shared by lipid $X$ and the LpxH substrate UDP-DAGn is recognized by an elaborate network of active site residues, which enables LpxH to differentiate its substrate UDPDAGn from an overwhelming amount of competing phospholipids in the bacterial inner membrane (Fig. 5C). The two acyl chains of lipid X are recognized differently. The $2-N$-linked acyl chain is buried inside a hydrophobic chamber formed between the core domain and the insertion lid, whereas the 3-O-linked acyl chain rises through an open area above the active site, with its remaining acyl chain interacting with surface-exposed hydrophobic residues of the insertion lid (Fig. 5D).

Incubation of UDP-DAGn with LpxH in $\mathrm{H}_{2}^{18} \mathrm{O}$ results in the cleavage of the pyrophosphate bond and incorporation of a solvent molecule into UMP, suggesting that the hydrolysis occurs through a $\mathrm{Mn}^{2+}$ activated water attacking the $\alpha$-phosphate of the UDP moiety (Fig. 5E) [65]. H. influenzae LpxH is most active at slightly alkaline $\mathrm{pH}$ values and exhibits a sharp decrease in its catalytic activity at acidic $\mathrm{pH}$ [72], consistent with the presence of a general base in the active site, most likely a catalytic histidine residue, with the pKa value of $\sim 6.6$ [72], 

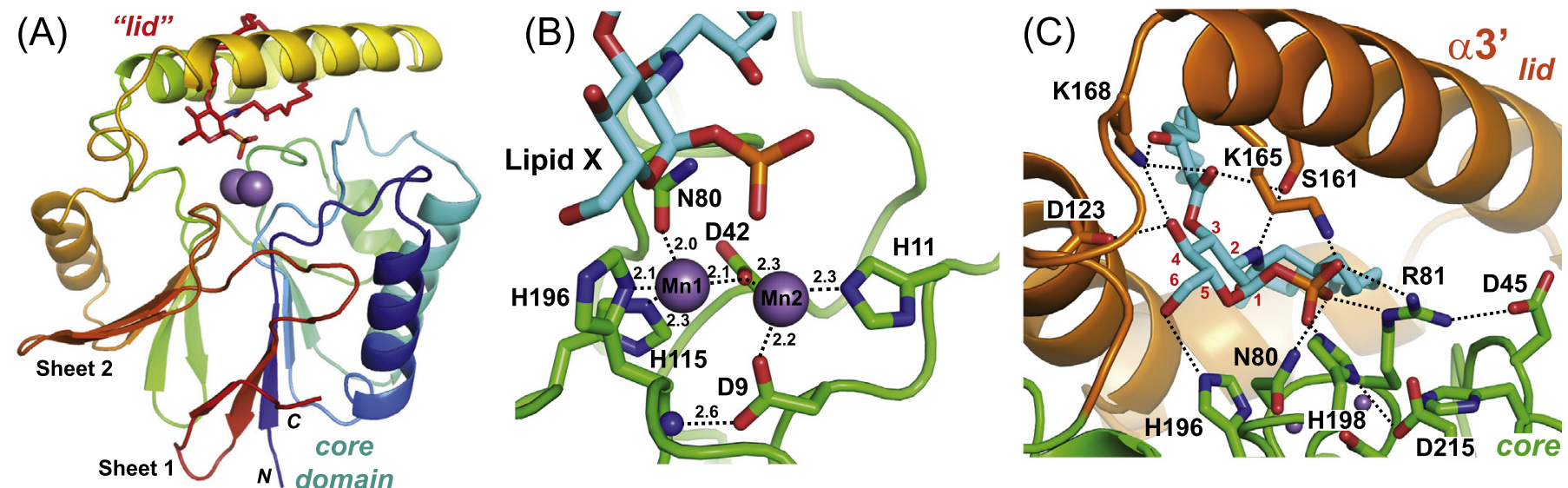

(D)

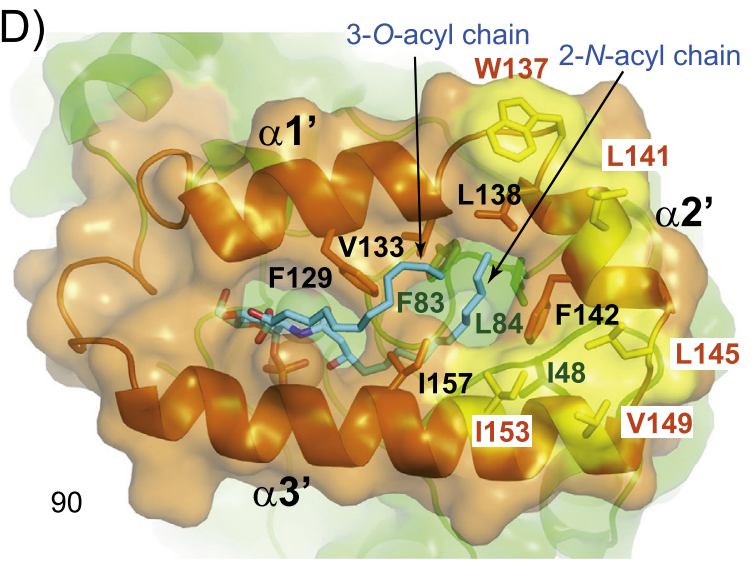

(F)

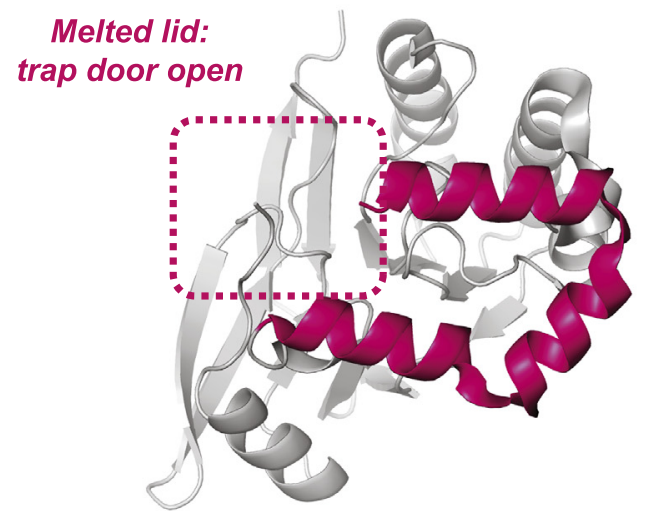

$(\mathrm{E})$
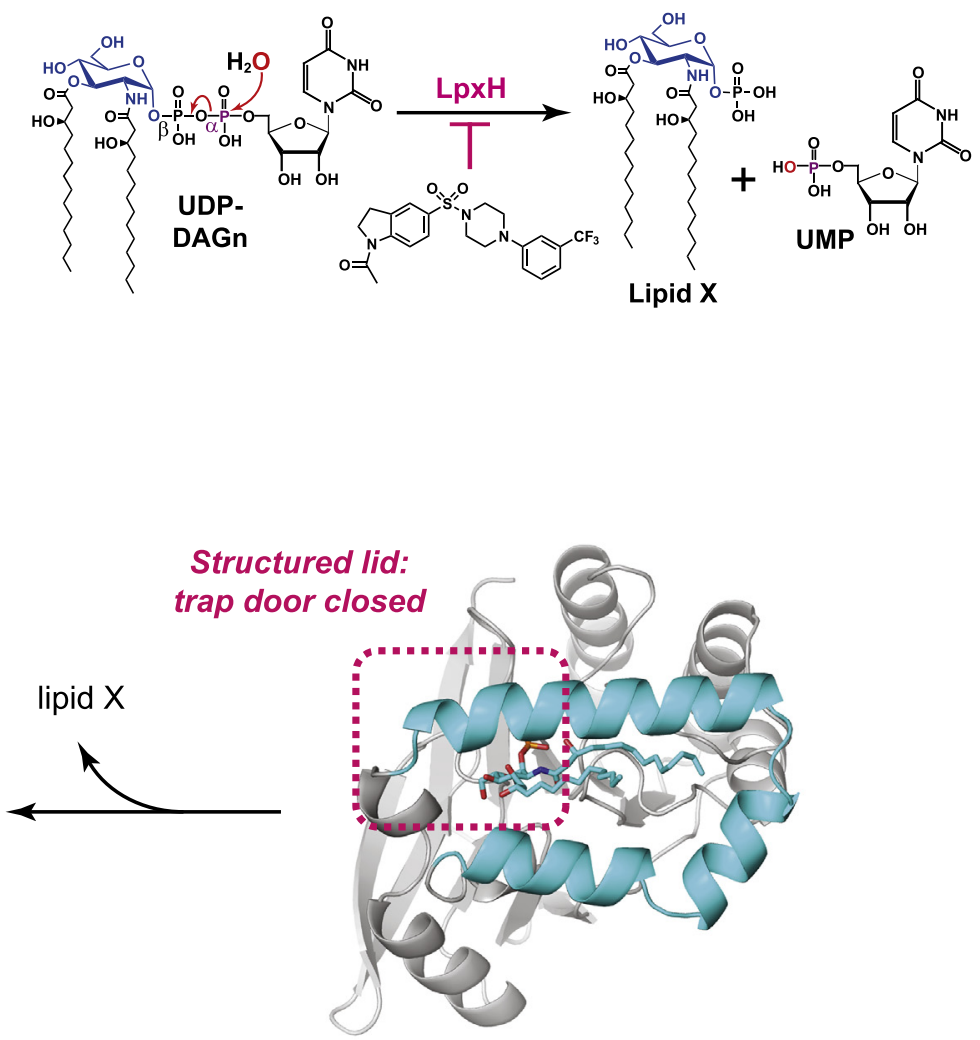

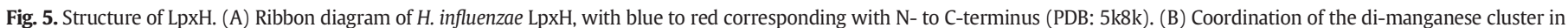

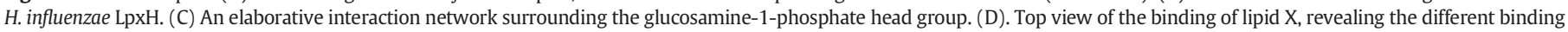

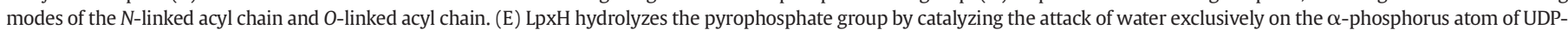

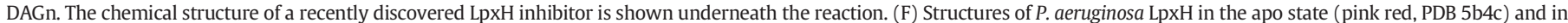
complex with lipid X (cyan, PDB 5b49) reveal a potential product release mechanism that involves the conformational melting of the insertion lid.

though such a pKa value may alternatively reflect a metal bound water molecule.

In parallel to the report of the H. influenzae LpxH structure occurred the structural elucidation of $P$. aeruginosa LpxH [74]. The P. aeruginosa LpxH structural analysis was carried out in the absence of any detergent, consistent with the notion that LpxH can exist both in the cytosolic and membrane-associated states. The structure of $P$. aeruginosa LpxH bound to the product lipid $\mathrm{X}$ is very similar to that of $H$. influenzae $\mathrm{LpxH}$, except that two $P$. aeruginosa LpxH molecules form a dimer to conceal the hydrophobic surface of the insertion lid, which likely mediates the membrane association of LpxH. Intriguingly, the manganese coordination in P. aeruginosa additionally involves H197 [74], whereas the corresponding $\mathrm{H} 198$ in $\mathrm{H}$. influenzae LpxH does not directly participate in manganese binding and has been suggested to serve as the catalytic general base [73]. As noted above, the pKa value of 6.6 for the catalytic base could reflect either an active site histidine or metal-bound water. Hence, whether H197 (P. aeruginosa LpxH)/H198 (H. influenzae LpxH) is solely involved in manganese coordination or actively participates 
in the catalysis requires additional biochemical analysis, for example, through comparative mutagenesis studies of individual manganese binding residues and their effect on the $\mathrm{pH}$ rate profile.

In addition to elucidating the structure of the $P$. aeruginosa LpxHlipid X complex, Okada and co-workers also captured the conformation of $P$. aeruginosa LpxH in the apo state [74]. Although such an analysis required the mutagenesis of a catalytically important, manganesecoordinating residue $(\mathrm{H} 10 \mathrm{~N})$ and the subsequent elimination of one of the two catalytic manganese ions, it nevertheless revealed a potential product release mechanism involving a partially melted insertion lid that may allow the release of lipid X through a trap door mechanism (Fig. 5F).

As LpxH is found in the vast majority of the clinically important Gram-negative pathogens, including $E$. coli, $H$. influenzae, $K$. pneumoniae, $S$. typhimurium, $V$. cholerae, $P$. aeruginosa, $N$. gonorrhoeae, A. baumannii, B. cenocepaciae, F. tularensis, and others, it is an important antibiotic target. Inhibition of LpxH results in accumulation of the toxic lipid intermediate, UDP-DAGn, and distortion of the bacterial inner membrane, which provides an independent mechanism of cell killing in addition to the general disruption of lipid A biosynthesis in Gram-negative bacteria [71,75].

In this regard, the recent discovery of a sulfonyl piperazine compound as a putative LpxH inhibitor was truly exciting (chemical structure shown in Fig. 5E) [76]. The compound displayed modest antibiotic activity ( $\mathrm{MIC}=0.25 \mu \mathrm{g} / \mathrm{mL}$ ) against an E. coli strain containing an efflux pump mutation $(\Delta t o l C)$. Spontaneous resistance mutations to this compound in E. coli were mapped exclusively to lpxH, and overexpression of LpxH resulted in an increased MIC. These results strongly indicate that the sulfonyl piperazine compound functions through inhibition of LpxH. Further biochemical and structural characterization of the mode of inhibition of this compound may ultimately enable the clinical development of LpxH-targeting antibiotics.

\section{2. $L p x I$}

A subset of Gram-negative organisms, including all $\alpha$-proteobacteria and diverse environmental isolates, lack LpxH. These bacteria nevertheless produce lipid A using the same set of enzymes upstream and downstream of the UDP-DAGn pyrophosphate hydrolase, suggesting the presence of one or more alternative enzymes that can catalyze the same hydrolase reaction of LpxH. The gene encoding such a function, IpxI, was identified based on its location between $\operatorname{lp} x A$ and $\operatorname{lp} x B$ in Caulobacter crescentus and was biochemically characterized in 2010 [77]. The structures of the enzyme in complex with the substrate and with the product were reported two years later [78]. These studies confirmed that LpxI is indeed a distinct enzyme from LpxH and the two enzymes bear no similarity either structurally or mechanistically $[66,78]$.

LpxI consists of an N-terminal lipid X-binding domain (LXD) and a Cterminal (LpxI) catalytic domain (ICD), which are connected by a short linker (Fig. 6A). The lipid binding domain contains a central $\beta$-sheet decorated by helices on both sides and lacks sequence homology to any other known proteins, whereas the catalytic domain shares structural similarity to cytosine deaminases. LpxI undergoes a large conformational switch during catalysis (Fig. 6A). In the UDP-DAGn-bound substrate complex, the catalytic domain is located adjacent to the lipid binding domain, and the two domains collectively recognize the UDPglucosamine head group and the two acyl chains of the substrate. In the product complex, however, the two domains swing apart; only the acyl chains of the product, lipid X, are recognized by the lipid binding domain, whereas the catalytic domain is unoccupied. These results, coupled with the observation that LpxI forms a dimer in solution and in the crystal structure, led to the proposal that the ligand-binding domain and the catalytic domain form halves of an inter-domain active site, and that the catalysis is accompanied by large scale conformational rearrangements triggered by substrate binding (Fig. 6B).
LpxI is also a metalloenzyme. In contrast to LpxH, which is selectively activated by $\mathrm{Mn}^{2+}$, LpxI can be activated both by $\mathrm{Mg}^{2+}$ and $\mathrm{Mn}^{2+}$, but it is most active in the presence of $\mathrm{Mg}^{2+}$ [77]. Intriguingly, incubation of UDP-DAGn with LpxI in $\mathrm{H}_{2}^{18} \mathrm{O}$ results in the incorporation of a solvent molecule into lipid $X$ instead of UMP, suggesting that a water molecule attacks the $\beta$-phosphate instead of the $\alpha$-phosphate as in the case of LpxH (Fig. 6C) [77]. Consistent with this observation, the catalytically important residue D225 (D225A in the substrate complex) is located closer to the $\beta$-phosphate group than the $\alpha$-phosphate group (Fig. 6A). Unfortunately, further insights into the molecular details of LpxI catalysis are hindered by the lack of the $\mathrm{Mg}^{2+}$ ion density in the active site of the substrate and would require further structural and mechanistic investigations.

\section{3. $L p x G$}

Even with the discovery of LpxH and LpxI, it was realized that Chlamydiae do not have an identifiable homolog of either of these two enzymes, despite the conservation of other lipid A enzymes. Our laboratory designed a complementation screen against the C. trachomatis genomic library using a conditional-lethal mutant of E. coli and identified a previously uncharacterized open reading frame (ORF)-C. trachomatis ORF 461 (ct461), renamed lpxG-as the third family of the UDP-DAGn pyrophosphatase [67]. Overexpression of LpxG results in toxic accumulation of lipid $X$ and profoundly reduces the infectivity of $C$. trachomatis, validating LpxG as the long-sought-after UDP-DAGn hydrolase. LpxG is highly conserved in Chlamydiae, and analysis of these LpxG orthologs reveals the presence of the signature metal-binding motifs found in the CLP family. However, it shows little sequence similarity to either LpxH or LpxI, highlighting LpxG as the founding member of a third class of UDP-DAGn hydrolases. The exceedingly low sequence identity of the unique UDP-DAGn pyrophosphatase LpxG compared with LpxH (11\%) and LpxI (9\%) forecasts distinct structural features within LpxG that could be exploited for developing highly specific antibiotics for treating Chlamydia infections without causing major alterations in resident microbial communities or leading to unintended antibiotic resistance among other co-infecting pathogens.

\section{LpxB}

LpxB, the lipid A disaccharide synthetase at the fifth step of the Raetz pathway (Fig. 1A), was one of the first two lipid A enzymes that were molecularly cloned and biochemically characterized (together with LpxA) in 1986 [5]. The complete sequence of E. coli lpxB gene (originally known as pgsB) was deciphered a year later [79].

LpxB is an inverting glycosyltransferase of family 19 of the GT-B superfamily (www.cazy.org) [77]. Like LpxH, LpxB is a membraneassociated lipid $A$ enzyme, and its catalysis requires the presence of detergents. LpxB is most active at neutral $\mathrm{pH}$, and its specific activity drops both under acidic and basic conditions [77]. Overexpression of LpxB in E. coli results in the accumulation of aberrant tubular membranes of a uniform diameter along the inner surface of the bacterial inner membrane, suggesting that the accumulation of the lipid product of the LpxB reaction, $2^{\prime}, 3^{\prime}$-diacylglucosamine- $\left(\beta, 1^{\prime}-6\right)-2,3-$ diacylglucosamine-1-phosphate (DSMP), is toxic to cells [77].

Apart from its role as the disaccharide synthetase to condense UDPDAGn with lipid X to form DSMP and UDP, little is known about LpxB, either mechanistically or structurally. Hence, the knowledge of the structure and mechanism of LpxB should greatly facilitate the development of novel antibiotics targeting this essential enzyme.

\section{LpxK}

LpxK is an integral membrane P-loop kinase that phosphorylates the 4'-hydroxyl group of DSMP to form tetraacyldisaccharide 1,4'bisphosphate (lipid $\mathrm{IV}_{\mathrm{A}}$ ) at the sixth step of the Raetz pathway 


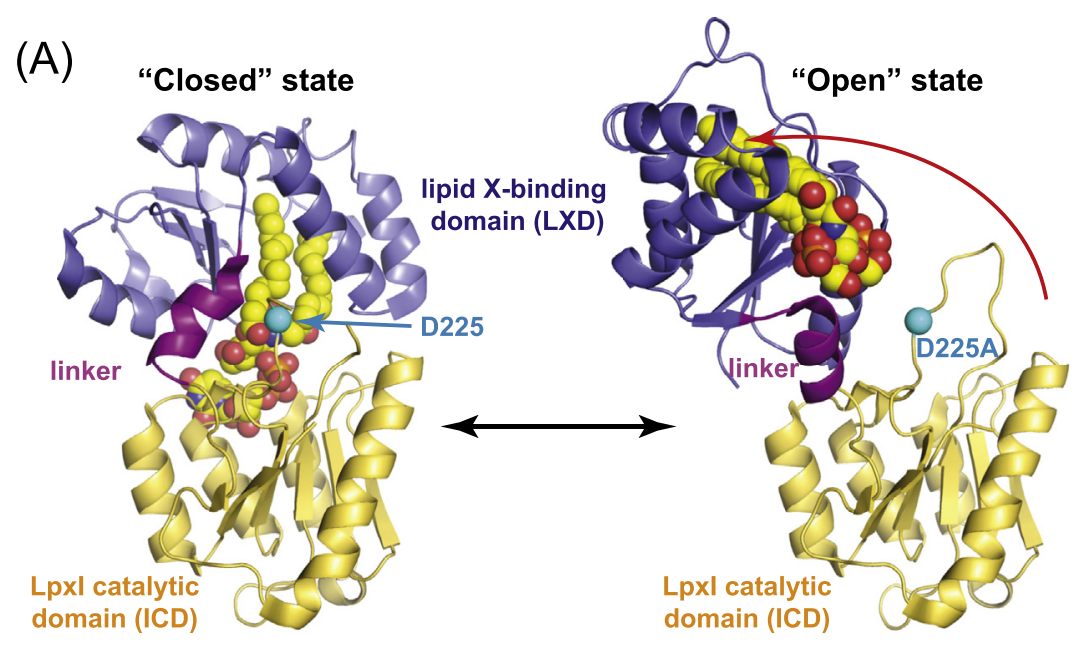

UDP-DAGn substrate complex

lipid X product complex

(B)

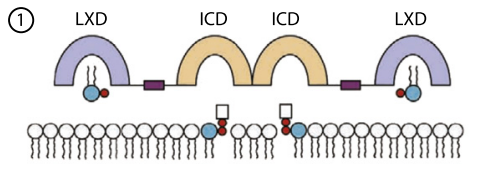

$\downarrow$

(2)

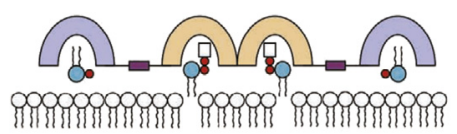

(3)

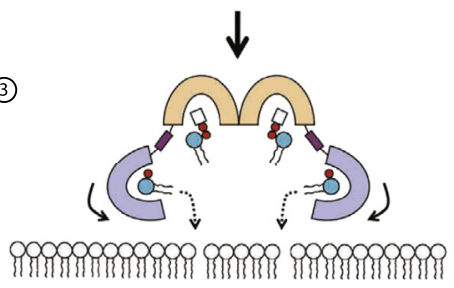

(6)

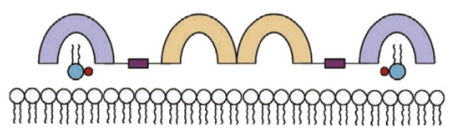

(5)

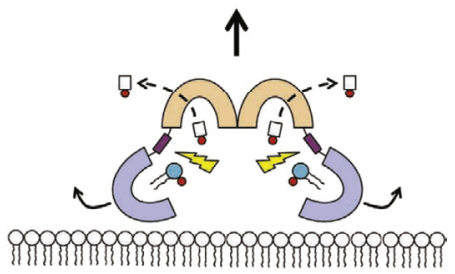

(4)

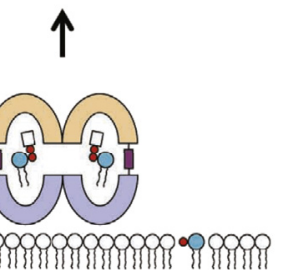

(C)
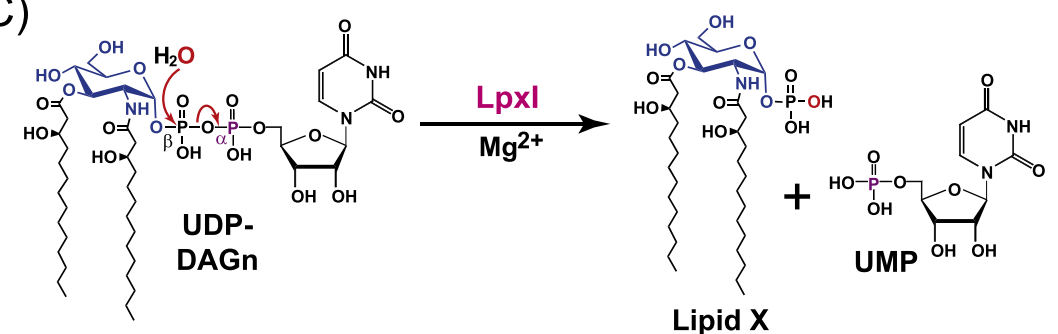

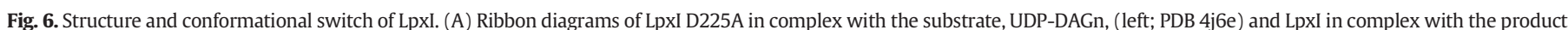

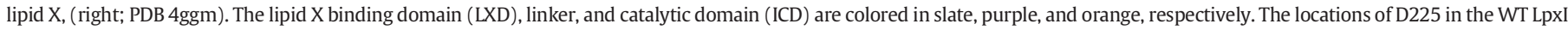

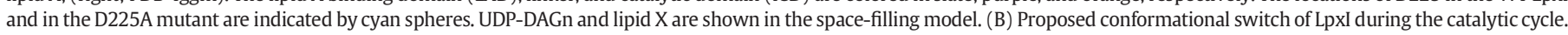
(C) LpxI hydrolyzes the pyrophosphate group by catalyzing the attack of water exclusively on the $\beta$-phosphorus atom of UDP-DAGn.

(Fig. 1A). The gene encoding LpxK was discovered in 1997 using expression cloning of the Kohara library [80]. Depletion of LpxK abolishes bacterial growth and results in accumulation of DSMP, verifying LpxK as an essential lipid kinase [81].

LpxK requires metal ions for catalysis and can be activated by $\mathrm{Mg}^{2+}$, $\mathrm{Mn}^{2+}$ and $\mathrm{Co}^{2+}[82]$. The highest activity of LpxK is achieved in the presence of $\mathrm{Mg}^{2+}$. Under the condition of equal molar ratio of ATP and $\mathrm{Mg}^{2+}$, steady state enzyme kinetics analysis supports the formation of a ternary LpxK-ATP/ $\mathrm{Mg}^{2+}$-DSMP complex [82] (Fig. 7A); however, whether the LpxK catalysis follows an ordered- or random-sequential bi-substrate mechanism requires additional investigations.

The structures of LpxK from A. aeolicus in the apo state and in complex with ADP/ $\mathrm{Mg}^{2+}$ were determined in 2012 [83], which were followed by structural analysis of LpxK in complex with the ATP analog, AMP- 
(A)

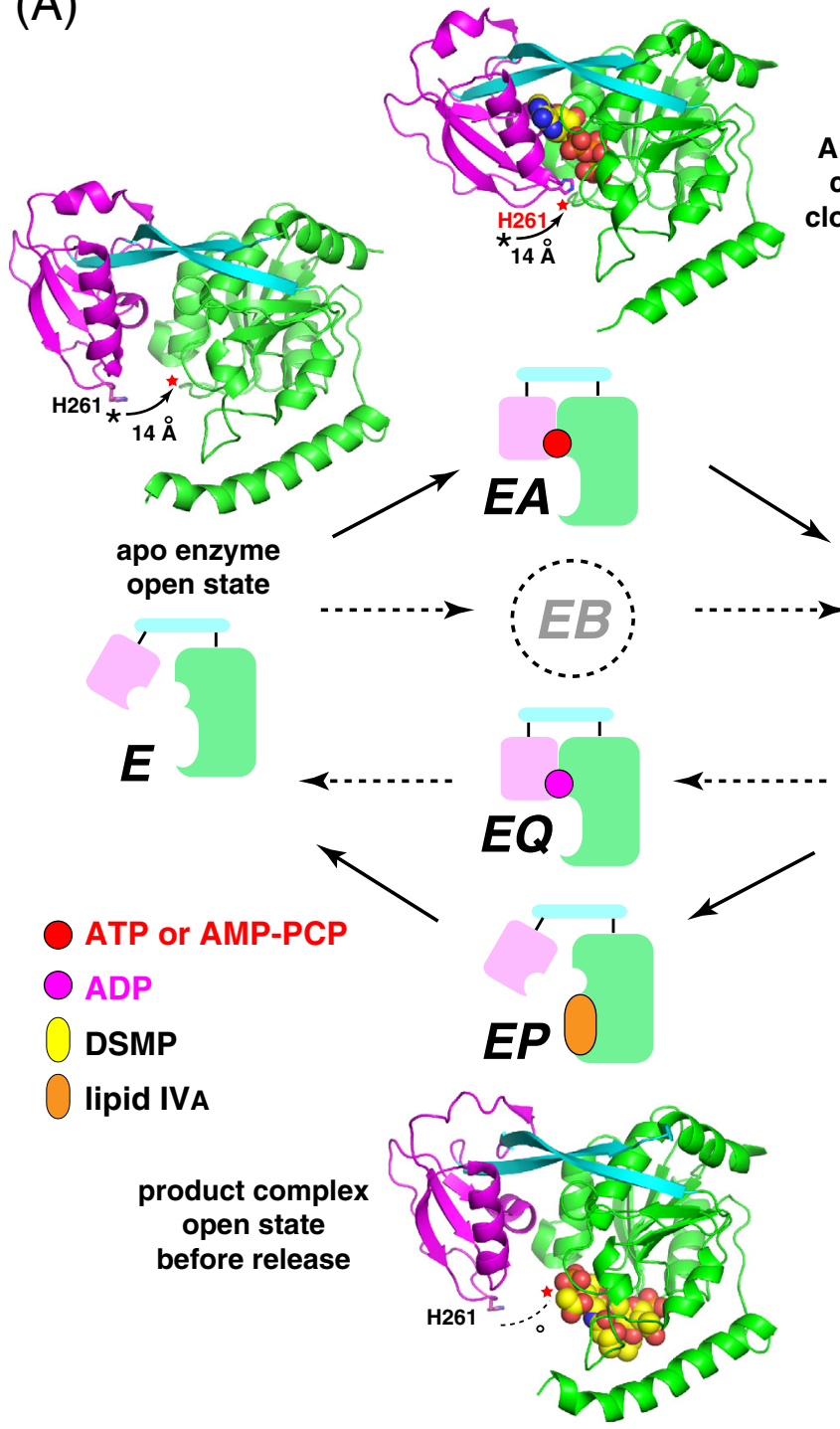

(B)

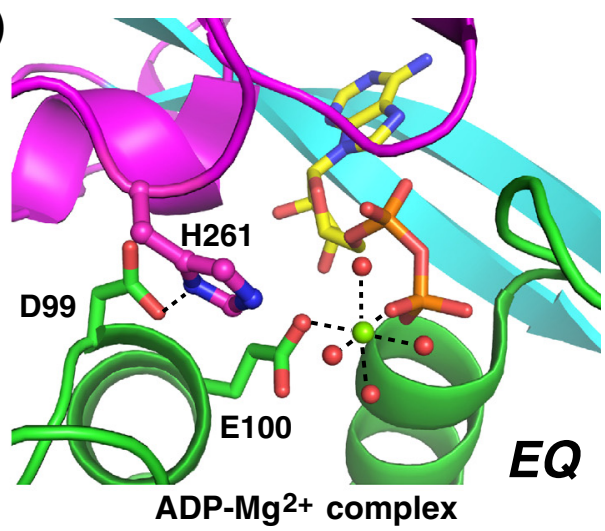

(C)

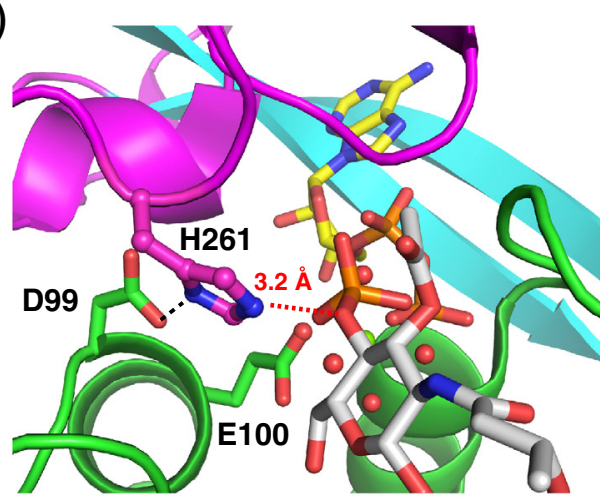

(D)

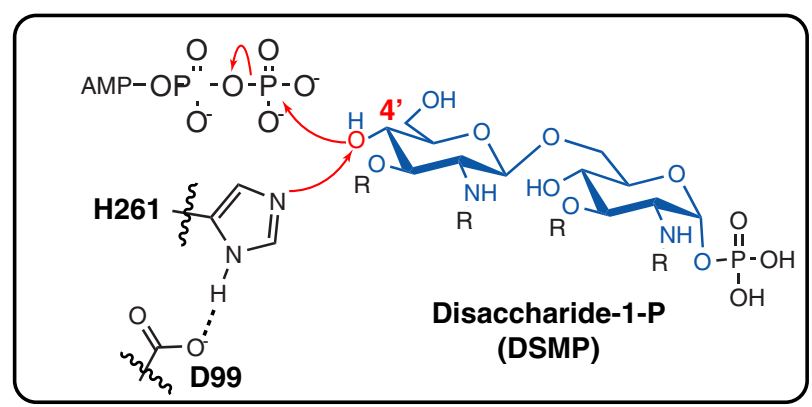

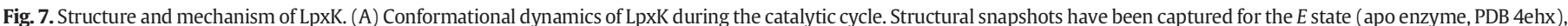

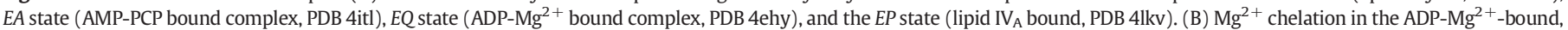

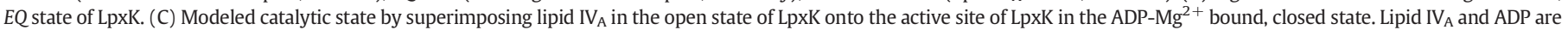
shown in the stick model with the carbon atoms of lipid $\mathrm{IV}_{\mathrm{A}}$ colored in grey and ADP colored in yellow, respectively. (D) The proposed mechanism of LpxK catalysis.

PCP [82], and the product lipid $\mathrm{IV}_{\mathrm{A}}$ [84]. LpxK possesses two Rossmannfold domains connected by a long linker consisting of two antiparallel $\beta$ strands (Fig. 7A). The larger $\mathrm{N}$-terminal domain (colored in green in Fig. 7A) consists of a central nine-stranded $\beta$-sheet that is flanked by seven $\alpha$-helices and a $3{ }_{10}$-helix; the smaller C-terminal domain (colored in purple in Fig. 7A) consists of a six-stranded $\beta$-sheet surrounded by two $\alpha$-helices and a $310^{-}$-helix. The ATP-binding chamber is formed at the interface of two domains (e.g., the red circle of the $E A$ state in Fig. 7A). In the apo state, the C-terminal domain is separated from the $\mathrm{N}$-terminal domain, creating an open ATP chamber. Binding of the ATP analog, AMP-PCP (EA state), or the product ADP/ $\mathrm{Mg}^{2+}$ (EQ state; Fig. 7B) induces a rotation of the $\mathrm{C}$-terminal domain around the linker, resulting in a movement of $\sim 14 \AA$ for $\mathrm{H} 261$ to form a hydrogen bond with D99 and generate the catalytic dyad, D99-H261. Such a movement also closes the ATP-binding chamber. In the ADP/ $\mathrm{Mg}^{2+}$ complex structure, the $\mathrm{Mg}^{2+}$ is coordinated by E100 of LpxK and an oxygen atom from the $\beta$-phosphate group of ADP (Fig. 7B). The remaining four ligands all come from solvent water molecules. Such a result suggests that $\mathrm{Mg}^{2+}$ binding may accompany the binding of ATP/ADP and that $\mathrm{Mg}^{2+}$ is released together with the release of ADP. However, binding of the ATP analog, AMP-PCP, has its $\gamma$-phosphate group occupying the $\mathrm{Mg}^{2+}$ binding site [82]. Whether this indicates a positional shift of the $\mathrm{Mg}^{2+}$ ion during catalysis [82] or a possible crystallization artifact due to the replacement of the phosphate oxygen atom by a carbon atom requires further structural and biochemical investigation.

The structure of the lipid $\mathrm{IV}_{\mathrm{A}}$ product complex has also been captured with LpxK [84]. Surprisingly, the structure reveals LpxK in an open conformational state, after releasing of ADP, but retaining the product lipid $\mathrm{IV}_{\mathrm{A}}$ (Fig. 7A, EP state). In this open state, the distance of $\mathrm{H} 261$ is over $14 \AA$ away from the active site; on the other hand, superimposing the coordinates of lipid $\mathrm{IV}_{\mathrm{A}}$ from the open EP state to LpxK in the ADP/ $\mathrm{Mg}^{2+}$-bound closed state has the H261 Ne1 atom within 3.2 A from the $4^{\prime}-\mathrm{O}$ atom of the product (Fig. 7C). Such a structural observation supports the proposal of D99-H261 as the catalytic dyad to 
activate the $4^{\prime}$-hydroxyl group of DSMP to attack the $\gamma$-phosphorus atom of ATP (Fig. 7D), a model that is corroborated by the observed pKa value of 6.6 for the catalytic general base [82]

LpxK is the last essential enzyme of the Raetz pathway. Although mutants lacking the next enzyme of the pathway, the Kdo-transferase KdtA, are viable upon overexpression of downstream transport proteins [85], no suppressor mutation has been isolated that confers viability to a knockout of the lpxK gene. Such an observation makes LpxK another excellent antibiotic target. Similar to LpxH inhibitors, inhibition of LpxK is expected to accumulate toxic lipid intermediates with a gross distortion of the bacterial inner membrane. Such a notion is also supported by a recent pathway analysis of lipid A enzymes [86]. In addition, great success has been achieved in developing kinase inhibitors for cancer therapy [87]. Lessons from these successes could be extended to the discovery of bacterial kinase inhibitors [88].

\section{Regulation of lipid A biosynthesis}

In order for Gram-negative bacteria to maintain the structural integrity of the inner membrane, the peptidoglycan layer, and the outer membrane, the biosynthesis of phospholipid, peptidoglycan, and lipid A must be coordinated. The regulatory network between peptidoglycan and lipid A biosyntheses is not well understood, except that one of the two starting materials of lipid A biosynthesis, UDP-GlcNAc, is shared with the peptidoglycan pathway (Fig. 8). On the other hand, accumulating evidence has started to unveil the intricate connections between the phospholipid and lipid A biosynthetic pathways.

As a regulatory enzyme catalyzing the committed step of lipid A biosynthesis, the modulation of LpxC has received much attention. It was noted that inhibition of LpxC can be compensated by mutations that compromise the activity of FabZ in the fatty acid biosynthetic pathway [89,90]; conversely, the LpxC level is notably lower in cells harboring the fabZ mutations [90]. On the other hand, overexpression of FabZ (and upregulation of phospholipid biosynthesis) is accompanied by an upregulation of the LpxC activity and vice versa $[90,91]$. As the biosynthesis of phospholipids depends on the production of fatty acids, these observations provide direct evidence that the biosynthetic pathways of lipid A and phospholipids are co-regulated.

LpxC is a substrate of the membrane protease FtsH [92], and the Cterminal tail of LpxC is required for the proteolytic processing [93]. The FtsH-dependent degradation of LpxC requires YciM [91]. Defects in either FtsH or YciM result in accumulation of LpxC and an enhanced lipid A-to-phospholipid ratio [91,92,94]. When yciM is present in multiple copies, there is a reduction in the LPS level, a phenomenon that is reversed by overexpression of LpxC [91]. Further characterization has revealed YciM as a heat shock protein and has led to its renaming as LapB (lipopolysaccharide assembly protein B) for its role in the assembly of LPS through interactions with LPS, FtsH, WaaC, and Lpt proteins from the LPS translocation complex [95].

How the proteolytic activity of FtsH is modulated is not well understood at the molecular level, but it may be downregulated by a specific form of acyl-ACPs or $\beta$-hydroxyacyl-ACPs, the central players in the biosyntheses of fatty acids and phospholipids [94]. Such a proposal is consistent with the observation of upregulation of LpxC by 5 - to 10 -fold when lipid A biosynthesis is inhibited [33], which presumably is caused by accumulation of phospholipids and acyl-ACP, and the subsequent suppression of the FtsH activity. It should be noted that in addition to regulating the level of LpxC, $\beta$-hydroxyacyl-ACP also directly participates in essential steps of lipid A biosynthesis: $\beta$-hydroxyacyl-ACP is a substrate of LpxA and LpxD at the first and third steps of lipid A biosynthesis. Therefore, accumulation of $\beta$-hydroxyacyl-ACP due to the overproduction of phospholipid may contribute to the upregulation of all first three essential steps of lipid A biosynthesis.

There have not been any detailed studies of potential regulatory mechanisms for the remaining three essential lipid A enzymes, LpxH/ LpxI/LpxG, LpxB and LpxK at the fourth, fifth and sixth steps of the

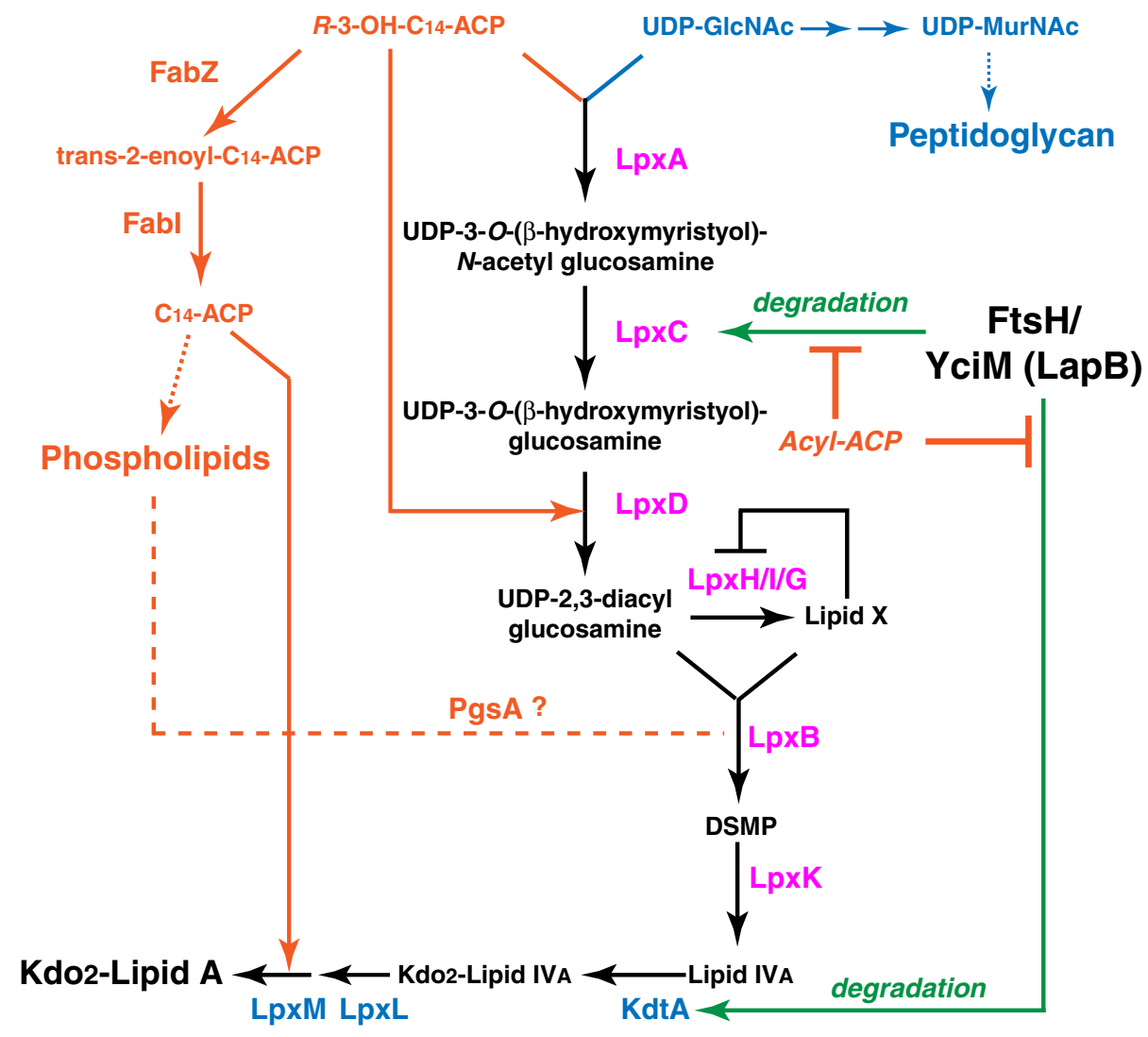

Fig. 8. Regulation of lipid A biosynthesis. 
Raetz pathway. The lipid A pyrophosphate hydrolases LpxH and LpxI copurify with the product lipid X $[73,78]$, suggesting a negative feedback loop due to product accumulation. Surprisingly, the di-saccharide synthetase LpxB has been reported to co-purify not with its product, DSMP, but instead with phospholipids [77], suggesting another possible regulatory node between lipid $A$ and phospholipid biosyntheses. In this case, LpxB may in turn contribute to the regulation of phospholipid biosynthesis. It was noted that the pgsA444 mutant of phosphatidylglycerophosphate synthetase (PgsA), an essential enzyme in the phosphatidylglycerol (PG) biosynthesis pathway, continues to make about two-thirds of the normal level of PG despite losing the majority of its in vitro activity [96]. Only with a second lesion at the $\operatorname{lp} x B$ locus, the double mutant displays a dramatically reduced PG level and becomes temperature sensitive [96]. Mutants with either lesion alone grow normally at all temperatures. Such an observation suggests a regulatory connection between the biosynthetic pathways of lipid A and phospholipids. Finally, although no mechanism has been reported for regulating LpxK at the sixth step of the Raetz pathway, the next enzyme of the pathway, the Kdo transferase $\mathrm{KdtA}$, is regulated by the membrane protease FtsH [97], again illustrating the co-regulatory nature of the lipid A and phospholipid biosynthetic pathways.

Recently, an elaborate regulatory network has been constructed that integrates existing biochemical evidence with mathematical modeling [98]. Experimental validation of these regulatory mechanisms and discovery of new contributing factors will undoubtedly enrich our understanding of the intricate connections between lipid A biosynthesis and phospholipid biosynthesis, allow a rational explanation of the levels of lipid A intermediates when the activities of individual lipid A enzymes are perturbed, and ultimately contribute to the development of novel antibiotics targeting the essential lipid A pathway against susceptible and multidrug-resistance Gram-negative pathogens.

\section{Conclusion}

The Raetz pathway of lipid A biosynthesis plays a vital role in the survival and fitness of Gram-negative bacteria in nature and in the human host. Research efforts in the past three decades have established the framework of the lipid A biosynthetic pathway. The molecular mechanisms underlying the regulation of lipid A biosynthesis and its coordination with the biosynthesis of phospholipids and peptidoglycans remain to be established. The first six enzymes of the Raetz pathway are essential and are attractive targets for the development of novel antibiotics. Further investigation of the structure, mechanism, and inhibition of these enzymes may ultimately enable the development of clinical therapeutics against susceptible and multidrug resistant Gram-negative pathogens by inhibiting lipid A biosynthesis.

\section{Transparency Document}

The Transparency document associated with this article can be found, in online version.

\section{Acknowledgment}

This work was supported by a grant from the National Institute of General Medical Sciences awarded to P.Z. (GM115355).

\section{References}

[1] H.C.J. Gram, Über die isolirte Färbung der Schizomyceten in Schnitt- und Trockenpräparaten, Fortschr. Med. 2 (1884) 185-189.

[2] K. Takayama, N. Qureshi, P. Mascagni, Complete structure of lipid A obtained from the lipopolysaccharides of the heptoseless mutant of Salmonella typhimurium, J. Biol. Chem. 258 (1983) 12801-12803.

[3] K. Takayama, N. Qureshi, P. Mascagni, M.A. Nashed, L. Anderson, C.R. Raetz, Fatty acyl derivatives of glucosamine 1-phosphate in Escherichia coli and their relation to lipid A. Complete structure of a diacyl GlcN-1-P found in a phosphatidylglycerol-deficient mutant, J. Biol. Chem. 258 (1983) 7379-7385.
4] M.S. Anderson, C.E. Bulawa, C.R. Raetz, The biosynthesis of Gram-negative endotoxin. Formation of lipid A precursors from UDP-GlcNAc in extracts of Escherichia coli, J. Biol. Chem. 260 (1985) 15536-15541.

[5] D.N. Crowell, M.S. Anderson, C.R. Raetz, Molecular cloning of the genes for lipid A disaccharide synthase and UDP-N-acetylglucosamine acyltransferase in Escherichia coli, J. Bacteriol. 168 (1986) 152-159.

[6] C.R.H. Raetz, C. Whitfield, Lipopolysaccharide endotoxins, Annu. Rev. Biochem. 71 (2002) 635-700

[7] C.R. Raetz, C.M. Reynolds, M.S. Trent, R.E. Bishop, Lipid A modification systems in Gram-negative bacteria, Annu. Rev. Biochem. 76 (2007) 295-329.

[8] C. Whitfield, M.S. Trent, Biosynthesis and export of bacterial lipopolysaccharides, Annu. Rev. Biochem. 83 (2014) 99-128.

[9] R.J. Bastidas, C.A. Elwell, J.N. Engel, R.H. Valdivia, Chlamydial intracellular survival strategies, Cold Spring Harb. Perspect. Med. 3 (2013) a010256.

[10] B.D. Nguyen, D. Cunningham, X. Liang, X. Chen, EJ. Toone, C.R. Raetz, P. Zhou, R.H. Valdivia, Lipooligosaccharide is required for the generation of infectious elementary bodies in Chlamydia trachomatis, Proc. Natl. Acad. Sci. U. S. A. 108 (2011) 10284-10289.

[11] J.H. Moffatt, M. Harper, P. Harrison, J.D. Hale, E. Vinogradov, T. Seemann, R. Henry, B. Crane, F. St Michael, A.D. Cox, B. Adler, R.L. Nation, J. Li, J.D. Boyce, Colistin resistance in Acinetobacter baumannii is mediated by complete loss of lipopolysaccharide production, Antimicrob. Agents Chemother. 54 (2010) 4971-4977.

[12] L. Lin, B. Tan, P. Pantapalangkoor, T. Ho, B. Baquir, A. Tomaras, J.I. Montgomery, U. Reilly, E.G. Barbacci, K. Hujer, R.A. Bonomo, L. Fernandez, R.E. Hancock, M.D. Adams, S.W. French, V.S. Buslon, B. Spellberg, Inhibition of LpxC protects mice from resistant Acinetobacter baumannii by modulating inflammation and enhancing phagocytosis, MBio 3 (2012)

[13] M. Garcia-Quintanilla, M. Carretero-Ledesma, P. Moreno-Martinez, R. Martin-Pena, J. Pachon, M.J. McConnell, Lipopolysaccharide loss produces partial colistin dependence and collateral sensitivity to azithromycin, rifampicin and vancomycin in Acinetobacter baumannii, Int. J. Antimicrob. Agents 46 (2015) 696-702.

[14] L. Steeghs, R. den Hartog, A. den Boer, B. Zomer, P. Roholl, P. van der Ley, Meningitis bacterium is viable without endotoxin, Nature 392 (1998) 449-450.

[15] D. Peng, W. Hong, B.P. Choudhury, R.W. Carlson, X.X. Gu, Moraxella catarrhalis bacterium without endotoxin, a potential vaccine candidate, Infect. Immun. 73 (2005) 7569-7577.

[16] C.R.H. Raetz, S.L. Roderick, A left-handed parallel beta helix in the structure of UDP$\mathrm{N}$-acetylglucosamine acyltransferase, Science 270 (1995) 997-1000.

[17] C.R. Sweet, S. Lin, R.J. Cotter, C.R. Raetz, A Chlamydia trachomatis UDP-Nacetylglucosamine acyltransferase selective for myristoyl-acyl carrier protein. Expression in Escherichia coli and formation of hybrid lipid A species, J. Biol. Chem. 276 (2001) 19565-19574.

[18] M.S. Anderson, H.G. Bull, S.M. Galloway, T.M. Kelly, S. Mohan, K. Radika, C.R.H. Raetz UDP- $N$-acetylglucosamine acyltransferase of Escherichia coli. The first step of endotoxin biosynthesis is thermodynamically unfavorable, J. Biol. Chem. 268 (1993) 19858-19865.

[19] N.L. Que-Gewirth, A.A. Ribeiro, S.R. Kalb, R.J. Cotter, D.M. Bulach, B. Adler, I.S. Girons, C. Werts, C.R. Raetz, A methylated phosphate group and four amide-linked acyl chains in Leptospira interrogans lipid A. The membrane anchor of an unusual lipopolysaccharide that activates TLR2, J. Biol. Chem. 279 (2004) 25420-25429.

[20] L.I. Robins, A.H. Williams, C.R. Raetz, Structural basis for the sugar nucleotide and acyl-chain selectivity of Leptospira interrogans LpxA, Biochemistry 48 (2009) 6191-6201.

[21] T.J. Wyckoff, S. Lin, R.J. Cotter, G.D. Dotson, C.R.H. Raetz, Hydrocarbon rulers in UDP$\mathrm{N}$-acetylglucosamine acyltransferases, J. Biol. Chem. 273 (1998) 32369-32372.

[22] A.H. Williams, C.R. Raetz, Structural basis for the acyl chain selectivity and mechanism of UDP- $\mathrm{N}$-acetylglucosamine acyltransferase, Proc. Natl. Acad. Sci. U. S. A. 104 (2007) 13543-13550.

[23] E.W. Smith, X. Zhang, C. Behzadi, L.D. Andrews, F. Cohen, Y. Chen, Structures of Pseudomonas aeruginosa LpxA reveal the basis for its substrate selectivity, Biochemistry 54 (2015) 5937-5948.

[24] T.J. Wyckoff, C.R.H. Raetz, The active site of Escherichia coli UDP-Nacetylglucosamine acyltransferase. Chemical modification and site-directed mutagenesis, J. Biol. Chem. 274 (1999) 27047-27055.

[25] A.H. Williams, R.M. Immormino, D.T. Gewirth, C.R. Raetz, Structure of UDP-Nacetylglucosamine acyltransferase with a bound antibacterial pentadecapeptide, Proc. Natl. Acad. Sci. U. S. A. 103 (2006) 10877-10882.

[26] R.J. Jenkins, G.D. Dotson, Dual targeting antibacterial peptide inhibitor of early lipid A biosynthesis, ACS Chem. Biol. 7 (2012) 1170-1177.

[27] R.E. Benson, E.B. Gottlin, D.J. Christensen, P.T. Hamilton, Intracellular expression of peptide fusions for demonstration of protein essentiality in bacteria, Antimicrob. Agents Chemother. 47 (2003) 2875-2881.

[28] S. Fernandez-Lopez, H.S. Kim, E.C. Choi, M. Delgado, J.R. Granja, A. Khasanov, K. Kraehenbuehl, G. Long, D.A. Weinberger, K.M. Wilcoxen, M.R. Ghadiri, Antibacterial agents based on the cyclic D,L-alpha-peptide architecture, Nature 412 (2001) 452-455.

[29] C.T. Walsh, Polyketide and nonribosomal peptide antibiotics: modularity and versatility, Science 303 (2004) 1805-1810.

[30] R.J. Jenkins, K.A. Heslip, J.L. Meagher, J.A. Stuckey, G.D. Dotson, Structural basis for the recognition of peptide RJPXD33 by acyltransferases in lipid A biosynthesis, J. Biol. Chem. 289 (2014) 15527-15535.

[31] B. Beall, J. Lutkenhaus, Sequence analysis, transcriptional organization, and insertional mutagenesis of the envA gene of Escherichia coli, J. Bacteriol. 169 (1987) 5408-5415.

[32] K. Young L.L. Silver, D. Bramhill, P. Cameron, S.S. Eveland, C.R.H. Raetz, S.A. Hyland, M.S. Anderson, The envA permeability/cell division gene of Escherichia coli encodes 
the second enzyme of lipid A biosynthesis. UDP-3-O-(R-3-hydroxymyristoyl)- $N$ acetylglucosamine deacetylase, J. Biol. Chem. 270 (1995) 30384-30391.

[33] P.G. Sorensen, J. Lutkenhaus, K. Young, S.S. Eveland, M.S. Anderson, C.R.H. Raetz, Regulation of UDP-3-O-[R-3-hydroxymyristoyl]- $N$-acetylglucosamine deacetylase in Escherichia coli. The second enzymatic step of lipid a biosynthesis, J. Biol. Chem. 271 (1996) 25898-25905.

[34] J.E. Jackman, C.R.H. Raetz, C.A. Fierke, UDP-3-O-(R-3-hydroxymyristoyl)-Nacetylglucosamine deacetylase of Escherichia coli is a zinc metalloenzyme, Biochemistry 38 (1999) 1902-1911.

[35] S.G. Gattis, M. Hernick, C.A. Fierke, Active site metal ion in UDP-3-O-((R)-3hydroxymyristoyl)- $N$-acetylglucosamine deacetylase (LpxC) switches between $\mathrm{Fe}(\mathrm{II})$ and $\mathrm{Zn}(\mathrm{II})$ depending on cellular conditions, J. Biol. Chem. 285 (2010) 33788-33796.

[36] M. Hernick, S.G. Gattis, J.E. Penner-Hahn, C.A. Fierke, Activation of Escherichia coli UDP-3-O- $\left[(R)\right.$-3-hydroxymyristoyl]-N-acetylglucosamine deacetylase by $\mathrm{Fe}^{2+}$ yields a more efficient enzyme with altered ligand affinity, Biochemistry 49 (2010) 2246-2255.

[37] B.E. Coggins, X. Li, A.L. McClerren, O. Hindsgaul, C.R.H. Raetz, P. Zhou, Structure of the LpxC deacetylase with a bound substrate-analog inhibitor, Nat. Struct. Biol. 10 (2003) 645-651.

[38] D.A. Whittington, K.M. Rusche, H. Shin, C.A. Fierke, D.W. Christianson, Crystal structure of LpxC, a zinc-dependent deacetylase essential for endotoxin biosynthesis, Proc. Natl. Acad. Sci. U. S. A. 100 (2003) 8146-8150.

[39] M.D. Miller, N. Gao, P.L. Ross, N.B. Olivier, Crystal structure of A. aeolicus LpxC with bound product suggests alternate deacetylation mechanism, Proteins 83 (2015) 1706-1719.

[40] B.E. Coggins, A.L. McClerren, L. Jiang, X. Li, J. Rudolph, O. Hindsgaul, C.R.H. Raetz, P. Zhou, Refined solution structure of the LpXC-TU-514 complex and pKa analysis of an active site histidine: insights into the mechanism and inhibitor design, Biochemistry 44 (2005) 1114-1126.

[41] A.L. McClerren, P. Zhou, Z. Guan, C.R.H. Raetz, J. Rudolph, Kinetic analysis of the zincdependent deacetylase in the lipid A biosynthetic pathway, Biochemistry 44 (2005) 1106-1113.

[42] M. Hernick, H.A. Gennadios, D.A. Whittington, K.M. Rusche, D.W. Christianson, C.A. Fierke, UDP-3-O-((R)-3-hydroxymyristoyl $)-N$-acetylglucosamine deacetylase functions through a general acid-base catalyst pair mechanism, J. Biol. Chem. 280 (2005) 16969-16978.

[43] M. Hernick, C.A. Fierke, Catalytic mechanism and molecular recognition of E. coli UDP-3-O-( $R$-3-Hydroxymyristoyl)- $N$-acetylglucosamine deacetylase probed by mutagenesis, Biochemistry 45 (2006) 15240-15248.

[44] G.M. Clayton, D.J. Klein, K.W. Rickert, S.B. Patel, M. Kornienko, J. Zugay-Murphy, J.C. Reid, S. Tummala, S. Sharma, S.B. Singh, L. Miesel, K.J. Lumb, S.M. Soisson, Structure of the bacterial deacetylase LpxC bound to the nucleotide reaction product reveals mechanisms of oxyanion stabilization and proton transfer, J. Biol. Chem. 288 (2013) 34073-34080.

[45] H.R. Onishi, B.A. Pelak, L.S. Gerckens, L.L. Silver, F.M. Kahan, M.H. Chen, A.A. Patchett, S.M. Galloway, S.A. Hyland, M.S. Anderson, C.R.H. Raetz, Antibacterial agents that inhibit lipid A biosynthesis, Science 274 (1996) 980-982.

[46] C.J. Lee, X. Liang, R. Gopalaswamy, J. Najeeb, E.D. Ark, E.J. Toone, P. Zhou, Structural basis of the promiscuous inhibitor susceptibility of Escherichia coli LpxC, ACS Chem. Biol. 9 (2014) 237-246.

[47] N.H. Anderson, J. Bowan, A. Erwin, E. Harvood, T. Kline, K. Mdluli, K.B. Pfister, R. Shawar, A. Wagman, A. Yabannavar, in: W.I.P. Organization (Ed.), Antibacterial agents, vol. WO 2004/062601 A2, Chiron, Emeryville, CA, 2004.

[48] A.L. McClerren, S. Endsley, J.L. Bowman, N.H. Andersen, Z. Guan, J. Rudolph, C.R. Raetz, A slow, tight-binding inhibitor of the zinc-dependent deacetylase LpxC of lipid A biosynthesis with antibiotic activity comparable to ciprofloxacin, Biochemistry 44 (2005) 16574-16583.

[49] C.J. Lee, X. Liang, X. Chen, D. Zeng, S.H. Joo, H.S. Chung, A.W. Barb, S.M. Swanson, R.A. Nicholas, Y. Li, E.J. Toone, C.R. Raetz, P. Zhou, Species-specific and inhibitordependent conformations of LpxC: implications for antibiotic design, Chem. Biol. 18 (2011) 38-47.

[50] X. Liang, C.J. Lee, X. Chen, H.S. Chung, D. Zeng, C.R. Raetz, Y. Li, P. Zhou, E.J. Toone, Syntheses, structures and antibiotic activities of LpxC inhibitors based on the diacetylene scaffold, Bioorg. Med. Chem. 19 (2011) 852-860.

[51] J.I. Montgomery, M.F. Brown, U. Reilly, L.M. Price, J.A. Abramite, J. Arcari, R. Barham, Y. Che, J.M. Chen, S.W. Chung, E.M. Collantes, C. Desbonnet, M. Doroski, J. Doty, J.J. Engtrakul, T.M. Harris, M. Huband, J.D. Knafels, K.L. Leach, S. Liu, A. Marfat, L. McAllister, E. McElroy, C.A. Menard, M. Mitton-Fry, L. Mullins, M.C. Noe, J. O'Donnell, R. Oliver, J. Penzien, M. Plummer, V. Shanmugasundaram, C. Thoma, A.P. Tomaras, D.P. Uccello, A. Vaz, D.G. Wishka, Pyridone methylsulfone hydroxamate LpxC inhibitors for the treatment of serious Gram-negative infections, J. Med. Chem. 55 (2012) 1662-1670.

[52] A.P. Tomaras, C.J. McPherson, M. Kuhn, A. Carifa, L. Mullins, D. George, C. Desbonnet, T.M. Eidem, J.I. Montgomery, M.F. Brown, U. Reilly, A.A. Miller, J.P. O'Donnell, LpxC inhibitors as new antibacterial agents and tools for studying regulation of lipid $\mathrm{A}$ biosynthesis in Gram-negative pathogens, MBio 5 (2014) (e01551-01514)

[53] C.J. Lee, X. Liang, Q. Wu, J. Najeeb, J. Zhao, R. Gopalaswamy, M. Titecat, F. Sebbane, N. Lemaitre, E.J. Toone, P. Zhou, Drug design from the cryptic inhibitor envelope, Nat. Commun. 7 (2016) 10638.

[54] M. Titecat, X. Liang, C.J. Lee, A. Charlet, D. Hocquet, T. Lambert, J.M. Pages, R. Courcol, F. Sebbane, E.J. Toone, P. Zhou, N. Lemaitre, High susceptibility of MDR and XDR Gramnegative pathogens to biphenyl-diacetylene-based difluoromethyl-allo-threonylhydroxamate LpxC inhibitors, J. Antimicrob. Chemother. 71 (2016) 2874-2882.

[55] I.B. Dicker, S. Seetharam, Cloning and nucleotide sequence of the firA gene and the firA200(Ts) allele from Escherichia coli, J. Bacteriol. 173 (1991) 334-344.
[56] I.B. Dicker, S. Seetharam, What is known about the structure and function of the Escherichia coli protein FirA? Mol. Microbiol. 6 (1992) 817-823.

[57] T.M. Kelly, S.A. Stachula, C.R. Raetz, M.S. Anderson, The firA gene of Escherichia coli encodes UDP-3-O-( $R$-3-hydroxymyristoyl)-glucosamine $N$-acyltransferase. The third step of endotoxin biosynthesis, J. Biol. Chem. 268 (1993) 19866-19874.

[58] L. Buetow, T.K. Smith, A. Dawson, S. Fyffe, W.N. Hunter, Structure and reactivity of LpxD, the $N$-acyltransferase of lipid A biosynthesis, Proc. Natl. Acad. Sci. U. S. A. 104 (2007) 4321-4326.

[59] C.M. Bartling, C.R. Raetz, Crystal structure and acyl chain selectivity of Escherichia col LpxD, the N-acyltransferase of lipid A biosynthesis, Biochemistry 48 (2009) 8672-8683.

[60] J. Badger, B. Chie-Leon, C. Logan, V. Sridhar, B. Sankaran, P.H. Zwart, V. Nienaber, The structure of LpxD from Pseudomonas aeruginosa at 1.3 Å resolution, Acta Crystallogr. Sect. F: Struct. Biol. Cryst. Commun. 67 (2011) 749-752.

[61] J. Badger, B. Chie-Leon, C. Logan, V. Sridhar, B. Sankaran, P.H. Zwart, V. Nienaber Structure determination of LpxD from the lipopolysaccharide-synthesis pathway of Acinetobacter baumannii, Acta Crystallogr. Sect. F: Struct. Biol. Cryst. Commun. 69 (2013) 6-9.

[62] C.M. Bartling, C.R. Raetz, Steady-state kinetics and mechanism of LpxD, the Nacyltransferase of lipid A biosynthesis, Biochemistry 47 (2008) 5290-5302.

[63] A. Masoudi, C.R. Raetz, P. Zhou, C.W.T. Pemble, Chasing acyl carrier protein through a catalytic cycle of lipid A production, Nature 505 (2014) 422-426.

[64] D.M. Byers, H. Gong, Acyl carrier protein: structure-function relationships in a conserved multifunctional protein family, Biochem. Cell Biol. 85 (2007) 649-662.

[65] K.J. Babinski, A.A. Ribeiro, C.R. Raetz, The Escherichia coli gene encoding the UDP-2,3diacylglucosamine pyrophosphatase of lipid A biosynthesis, J. Biol. Chem. 277 (2002) 25937-25946

[66] L.E.T. Metzger, C.R. Raetz, An alternative route for UDP-diacylglucosamine hydrolysis in bacterial lipid A biosynthesis, Biochemistry 49 (2010) 6715-6726.

[67] H.E. Young, J. Zhao, J.R. Barker, Z. Guan, R.H. Valdivia, P. Zhou, Discovery of the elusive UDP-diacylglucosamine hydrolase in the lipid A biosynthetic pathway in Chlamydia trachomatis, MBio 7 (2016).

[68] T. Icho, C.E. Bulawa, C.R. Raetz, Molecular cloning and sequencing of the gene for CDP-diglyceride hydrolase of Escherichia coli, J. Biol. Chem. 260 (1985) 12092-12098.

[69] C.E. Bulawa, C.R. Raetz, Isolation and characterization of Escherichia coli strains defective in CDP-diglyceride hydrolase, J. Biol. Chem. 259 (1984) 11257-11264.

[70] Y. Kohara, K. Akiyama, K. Isono, The physical map of the whole E. coli chromosome: application of a new strategy for rapid analysis and sorting of a large genomic library, Cell 50 (1987) 495-508.

[71] K.J. Babinski, S.J. Kanjilal, C.R. Raetz, Accumulation of the lipid A precursor UDP-2,3diacylglucosamine in an Escherichia coli mutant lacking the lpxH gene, J. Biol. Chem 277 (2002) 25947-25956.

[72] H.E. Young, M.P. Donohue, T.I. Smirnova, A.I. Smirnov, P. Zhou, The UDPdiacylglucosamine pyrophosphohydrolase LpxH in lipid A biosynthesis utilizes $\mathrm{Mn}^{2+}$ cluster for catalysis, J. Biol. Chem. 288 (2013) 26987-27001.

[73] J. Cho, C.J. Lee, J. Zhao, H.E. Young, P. Zhou, Structure of the essential Haemophilus influenzae UDP-diacylglucosamine pyrophosphohydrolase LpxH in lipid A biosynthesis, Nat. Microbiol. 1 (2016) 16154.

[74] C. Okada, H. Wakabayashi, M. Kobayashi, A. Shinoda, I. Tanaka, M. Yao, Crystal structures of the UDP-diacylglucosamine pyrophosphohydrase LpxH from Pseudomonas aeruginosa, Sci. Rep. 6 (2016) 32822.

[75] D.L. Richie, K.T. Takeoka, J. Bojkovic, L.E.T. Metzger, C.M. Rath, W.S. Sawyer, J.R. Wei C.R. Dean, Toxic Accumulation of LPS Pathway Intermediates Underlies the Requirement of LpxH for Growth of Acinetobacter baumannii ATCC 19606, PLoS One 11 (2016) e0160918.

[76] A.S. Nayar, T.J. Dougherty, K.E. Ferguson, B.A. Granger, L. McWilliams, C. Stacey, L.J. Leach, S. Narita, H. Tokuda, A.A. Miller, D.G. Brown, S.M. McLeod, Novel antibacterial targets and compounds revealed by a high-throughput cell wall reporter assay, J. Bacteriol. 197 (2015) 1726-1734.

[77] L.E.T. Metzger, C.R. Raetz, Purification and characterization of the lipid A disaccharide synthase (LpxB) from Escherichia coli, a peripheral membrane protein, Biochemistry 48 (2009) 11559-11571.

[78] L.E.T. Metzger, J.K. Lee, J.S. Finer-Moore, C.R. Raetz, R.M. Stroud, LpxI structures reveal how a lipid A precursor is synthesized, Nat. Struct. Mol. Biol. 19 (2012) $1132-1138$.

[79] D.N. Crowell, W.S. Reznikoff, C.R. Raetz, Nucleotide sequence of the Escherichia coli gene for lipid A disaccharide synthase, J. Bacteriol. 169 (1987) 5727-5734.

[80] T.A. Garrett, J.L. Kadrmas, C.R. Raetz, Identification of the gene encoding the Escherichia coli lipid A 4'-kinase. Facile phosphorylation of endotoxin analogs with recombinant LpxK, J. Biol. Chem. 272 (1997) 21855-21864.

[81] T.A. Garrett, N.L. Que, C.R. Raetz, Accumulation of a lipid A precursor lacking the 4' phosphate following inactivation of the Escherichia coli lpxK gene, J. Biol. Chem. 273 (1998) 12457-12465

[82] R.P. Emptage, C.W.T. Pemble, J.D. York, C.R. Raetz, P. Zhou, Mechanistic characterization of the tetraacyldisaccharide-1-phosphate $4^{\prime}$-kinase LpxK involved in lipid A biosynthesis, Biochemistry 52 (2013) 2280-2290.

[83] R.P. Emptage, K.D. Daughtry, C.W.T. Pemble, C.R. Raetz, Crystal structure of LpxK, the 4'-kinase of lipid A biosynthesis and atypical P-loop kinase functioning at the membrane interface, Proc. Natl. Acad. Sci. U. S. A. 109 (2012) 12956-12961.

[84] R.P. Emptage, N.K. Tonthat, J.D. York, M.A. Schumacher, P. Zhou, Structural basis of lipid binding for the membrane-embedded tetraacyldisaccharide-1-phosphate $4^{\prime}$ kinase LpxK, J. Biol. Chem. 289 (2014) 24059-24068.

[85] C.M. Reynolds, C.R. Raetz, Replacement of lipopolysaccharide with free lipid A molecules in Escherichia coli mutants lacking all core sugars, Biochemistry 48 (2009) 9627-9640. 
[86] A. Emiola, J. George, S.S. Andrews, A complete pathway model for lipid A biosynthesis in Escherichia coli, PLoS One 10 (2015) e0121216.

[87] P. Wu, T.E. Nielsen, M.H. Clausen, FDA-approved small-molecule kinase inhibitors, Trends Pharmacol. Sci. 36 (2015) 422-439.

[88] C.T. Walsh, T.A. Wencewicz, Prospects for new antibiotics: a molecule-centered perspective, J. Antibiot. 67 (2014) 7-22.

[89] J.M. Clements, F. Coignard, I. Johnson, S. Chandler, S. Palan, A. Waller, J. Wijkmans, M.G. Hunter, Antibacterial activities and characterization of novel inhibitors of LpxC, Antimicrob. Agents Chemother. 46 (2002) 1793-1799.

[90] D. Zeng, J. Zhao, H.S. Chung, Z. Guan, C.R. Raetz, P. Zhou, Mutants resistant to LpxC inhibitors by rebalancing cellular homeostasis, J. Biol. Chem. 288 (2013) 5475-5486.

[91] S. Mahalakshmi, M.R. Sunayana, L. SaiSree, M. Reddy, yciM is an essential gene required for regulation of lipopolysaccharide synthesis in Escherichia coli, Mol. Microbiol. 91 (2014) 145-157.

[92] T. Inagawa, J. Kato, H. Niki, K. Karata, T. Ogura, Defective plasmid partition in ftsH mutants of Escherichia coli, Mol. Gen. Genomics. 265 (2001) 755-762.

[93] F. Fuhrer, A. Muller, H. Baumann, S. Langklotz, B. Kutscher, F. Narberhaus, Sequence and length recognition of the C-terminal turnover element of LpxC, a soluble substrate of the membrane-bound FtsH protease, J. Mol. Biol. 372 (2007) 485-496.
[94] T. Ogura, K. Inoue, T. Tatsuta, T. Suzaki, K. Karata, K. Young L.H. Su, C.A. Fierke, J.E. Jackman, C.R. Raetz, J. Coleman, T. Tomoyasu, H. Matsuzawa, Balanced biosynthesis of major membrane components through regulated degradation of the committed enzyme of lipid A biosynthesis by the AAA protease FtsH (HflB) in Escherichia coli, Mol. Microbiol. 31 (1999) 833-844.

[95] G. Klein, N. Kobylak, B. Lindner, A. Stupak, S. Raina, Assembly of lipopolysaccharide in Escherichia coli requires the essential LapB heat shock protein, J. Biol. Chem. 289 (2014) 14829-14853.

[96] M. Nishijima, C.E. Bulawa, C.R. Raetz, Two interacting mutations causing temperature-sensitive phosphatidylglycerol synthesis in Escherichia coli membranes, J. Bacteriol. 145 (1981) 113-121.

[97] C. Katz, E.Z. Ron, Dual role of FtsH in regulating lipopolysaccharide biosynthesis in Escherichia coli, J. Bacteriol. 190 (2008) 7117-7122.

[98] A. Emiola, S.S. Andrews, C. Heller, J. George, Crosstalk between the lipopolysaccharide and phospholipid pathways during outer membrane biogenesis in Escherichia coli, Proc. Natl. Acad. Sci. U. S. A. 113 (2016) 3108-3113. 